\title{
Measurement of azimuthal asymmetries associated with deeply virtual Compton scattering on an unpolarized deuterium target
}

The HERMES Collaboration

A. Airapetian ${ }^{\ell, o}$ N. Akopov ${ }^{\mathrm{Z}}$ Z. Akopov ${ }^{\mathrm{e}}$ M. Amarian ${ }^{\mathrm{f}, 1}$

E.C. Aschenauer ${ }^{\mathrm{f}, 2}$ W. Augustyniak ${ }^{\mathrm{y}}$ R. Avakian ${ }^{\mathrm{z}}$ A. Avetissian ${ }^{\mathrm{Z}}$

E. Avetisyan ${ }^{e}$ B. Ball ${ }^{\circ}$ S. Belostotski ${ }^{r}$ N. Bianchi ${ }^{j}$ H.P. Blok ${ }^{q, x}$

H. Böttcher ${ }^{f}$ A. Borissov ${ }^{e}$ J. Bowles ${ }^{m}$ V. Bryzgalov ${ }^{\mathrm{s}}$ J. Burns ${ }^{\mathrm{m}}$

M. Capiluppi ${ }^{i}$ G.P. Capitani ${ }^{j}$ E. Cisbani ${ }^{u}$ G. Ciullo ${ }^{i}$ M. Contalbrigo ${ }^{i}$

P.F. Dalpiaz ${ }^{i}$ W. Deconinck ${ }^{e, o, 3}$ R. De Leo ${ }^{b}$ L. De Nardo ${ }^{e, v}$

E. De Sanctis ${ }^{j}$ M. Diefenthaler ${ }^{h}$ P. Di Nezza ${ }^{j}$ J. Dreschler ${ }^{q}$ M. Düren ${ }^{\ell}$

M. Ehrenfried ${ }^{\ell, 4}$ G. Elbakian ${ }^{z}$ F. Ellinghaus ${ }^{d, 5}$ R. Fabbri ${ }^{\mathrm{f}}$ A. Fantoni ${ }^{\mathrm{j}}$

L. Felawka ${ }^{\mathrm{v}}$ S. Frullani ${ }^{\mathrm{u}}$ D. Gabbert ${ }^{\mathrm{f}}$ G. Gapienko ${ }^{\mathrm{s}}$ V. Gapienko ${ }^{\mathrm{s}}$

F. Garibaldi ${ }^{u}$ G. Gavrilov ${ }^{\mathrm{e}, \mathrm{r}, \mathrm{v}}$ V. Gharibyan ${ }^{\mathrm{z}}$ F. Giordano ${ }^{\mathrm{e}, \mathrm{i}}$ S. Gliske ${ }^{\circ}$ C. Hadjidakis ${ }^{\mathrm{j}, 6}$ M. Hartig ${ }^{\mathrm{e}, 7}$ D. Hasch $^{\mathrm{j}}$ T. Hasegawa ${ }^{\mathrm{w}}$ G. Hill ${ }^{\mathrm{m}}$

A. Hillenbrand ${ }^{\mathrm{f}}$ M. Hoek ${ }^{\mathrm{m}}$ Y. Holler ${ }^{\mathrm{e}}$ I. Hristova $^{\mathrm{f}}{ }^{\mathrm{Y}}$. Imazu ${ }^{\mathrm{w}}$

A. Ivanilov ${ }^{\mathrm{s}}$ A. Izotov ${ }^{\mathrm{r}}$ H.E. Jackson ${ }^{\mathrm{a}}$ A. Jgoun ${ }^{\mathrm{r}}$ H.S. Jo ${ }^{\mathrm{k}}$ S. Joosten ${ }^{\mathrm{n}, \mathrm{k}}$

R. Kaiser ${ }^{\mathrm{m}}$ G. Karyan ${ }^{\mathrm{z}}$ T. Keri ${ }^{\mathrm{m}, \ell}$ E. Kinney ${ }^{\mathrm{d}}$ A. Kisselev ${ }^{\mathrm{r}}$

N. Kobayashi ${ }^{w}$ V. Korotkov ${ }^{\mathrm{s}}$ V. Kozlov ${ }^{\mathrm{p}}$ B. Krauss ${ }^{\mathrm{h}, 8}$ P. Kravchenko ${ }^{\mathrm{r}}$ V.G. Krivokhijine ${ }^{\mathrm{g}}$ L. Lagamba $^{\mathrm{b}}$ R. Lamb ${ }^{\mathrm{n}}$ L. Lapikás $^{\mathrm{q}}$ I. Lehmann ${ }^{\mathrm{m}}$ P. Lenisa ${ }^{i}$ L.A. Linden-Levy ${ }^{n}$ A. López Ruiz ${ }^{k}$ W. Lorenzon ${ }^{\circ}$ X.-G. Lu ${ }^{\mathrm{f}}$ X.-R. Lu ${ }^{\mathrm{w}, 9}$ B.-Q. $\mathrm{Ma}^{\mathrm{c}}$ D. Mahon ${ }^{\mathrm{m}}$ N.C.R. Makins ${ }^{\mathrm{n}}$ S.I. Manaenkov ${ }^{\mathrm{r}}$

L. Manfré ${ }^{u}$ Y. Mao ${ }^{c}$ B. Marianski ${ }^{y}$ A. Martinez de la Ossa ${ }^{d}$

H. Marukyan ${ }^{z}$ C.A. Miller ${ }^{v}$ Y. Miyachi ${ }^{w}$ A. Movsisyan ${ }^{z}$ V. Muccifora ${ }^{j}$

D. Müller ${ }^{10}$ M. Murray ${ }^{\mathrm{m}}$ A. Mussgiller ${ }^{\mathrm{e}, \mathrm{h}}$ E. Nappi ${ }^{\mathrm{b}}$ Y. Naryshkin ${ }^{\mathrm{r}}$

A. Nass ${ }^{h}$ M. Negodaev ${ }^{f}$ W.-D. Nowak ${ }^{f}$ L.L. Pappalardo ${ }^{i}$

R. Perez-Benito ${ }^{\ell}$ N. Pickert ${ }^{\text {h, } 8}$ M. Raithel ${ }^{\text {h }}$ P.E. Reimer ${ }^{\mathrm{a}}$ A.R. Reolon ${ }^{\mathrm{j}}$

C. Riedl ${ }^{\mathrm{f}}$ K. Rith ${ }^{\mathrm{h}}$ G. Rosner ${ }^{\mathrm{m}}$ A. Rostomyan ${ }^{\mathrm{e}}$ J. Rubin ${ }^{\mathrm{n}}$ D. Ryckbosch ${ }^{\mathrm{k}}$

Y. Salomatin ${ }^{\mathrm{s}}$ F. Sanftl ${ }^{\mathrm{t}}$ A. Schäfer ${ }^{\mathrm{t}}$ G. Schnell ${ }^{\mathrm{f}, \mathrm{k}}$ K.P. Schüler ${ }^{\mathrm{e}}$

B. Seitz ${ }^{m}$ T.-A. Shibata ${ }^{\text {w }}$ V. Shutov ${ }^{g}$ M. Stancari ${ }^{i}$ M. Statera ${ }^{\mathrm{i}}$ 
E. Steffens ${ }^{\text {h }}$ J.J.M. Steijger ${ }^{q}$ H. Stenzel ${ }^{\ell}$ J. Stewart ${ }^{\text {f,2 }}$ F. Stinzing ${ }^{\text {h }}$

S. Taroian ${ }^{\mathrm{z}}$ A. Terkulov ${ }^{\mathrm{p}}$ A. Trzcinski ${ }^{\mathrm{y}}$ M. Tytgat ${ }^{\mathrm{k}}$ A. Vandenbroucke ${ }^{\mathrm{k}, 11}$

P.B. Van der Nat ${ }^{q}$ Y. Van Haarlem ${ }^{\mathrm{k}, 12}$ C. Van Hulse ${ }^{\mathrm{k}}$ M. Varanda ${ }^{\mathrm{e}}$

D. Veretennikov ${ }^{\mathrm{r}}$ V. Vikhrov ${ }^{\mathrm{r}}$ I. Vilardi ${ }^{\mathrm{b}, 13}$ C. Vogel ${ }^{\mathrm{h}, 14}$ S. Wang ${ }^{\mathrm{c}}$ S. Yaschenko ${ }^{\text {f,h }}$ H. Ye ${ }^{\mathrm{c}}$ Z. Ye ${ }^{\mathrm{e}, 15} \mathrm{~S}^{\mathrm{S}} \mathrm{Yen}^{\mathrm{v}}$ W. Yu ${ }^{\ell}$ D. Zeiler ${ }^{\mathrm{h}}$ B. Zihlmann ${ }^{\mathrm{e}, 16}$ P. Zupranski ${ }^{\mathrm{y}}$

${ }^{a}$ Physics Division, Argonne National Laboratory, Argonne, Illinois 60439-4843, USA

${ }^{\mathrm{b}}$ Istituto Nazionale di Fisica Nucleare, Sezione di Bari, 70124 Bari,Italy

${ }^{\mathrm{c}}$ School of Physics, Peking University, Beijing 100871, China

d Nuclear Physics Laboratory, University of Colorado, Boulder, Colorado 80309-0390, USA e DESY, 22603 Hamburg, Germany

${ }^{\mathrm{f}} D E S Y, 15738$ Zeuthen, Germany

$\mathrm{g}$ Joint Institute for Nuclear Research, 141980 Dubna, Russia

${ }^{\mathrm{h}}$ Physikalisches Institut, Universität Erlangen-Nürnberg, 91058 Erlangen, Germany

${ }^{\mathrm{i}}$ Istituto Nazionale di Fisica Nucleare, Sezione di Ferrara and Dipartimento di Fisica, Università di Ferrara, 44100 Ferrara, Italy

j Istituto Nazionale di Fisica Nucleare, Laboratori Nazionali di Frascati, 00044 Frascati, Italy

${ }^{\mathrm{k}}$ Department of Subatomic and Radiation Physics, University of Gent, 9000 Gent, Belgium

${ }^{\ell}$ Physikalisches Institut, Universität Gießen, 35392 Gießen, Germany

m Department of Physics and Astronomy, University of Glasgow, Glasgow G12 8QQ, United Kingdom

${ }^{\mathrm{n}}$ Department of Physics, University of Illinois, Urbana, Illinois 61801-3080, USA

${ }^{\circ}$ Randall Laboratory of Physics, University of Michigan, Ann Arbor, Michigan 48109-1040, USA

${ }^{\mathrm{P}}$ Lebedev Physical Institute, 117924 Moscow, Russia

q National Institute for Subatomic Physics (Nikhef), 1009 DB Amsterdam, The Netherlands

${ }^{\mathrm{r}}$ Petersburg Nuclear Physics Institute, Gatchina, Leningrad region, 188300 Russia

${ }^{\mathrm{s}}$ Institute for High Energy Physics, Protvino, Moscow region, 142281 Russia

${ }^{\mathrm{t}}$ Institut für Theoretische Physik, Universität Regensburg, 93040 Regensburg, Germany

${ }^{\mathrm{u}}$ Istituto Nazionale di Fisica Nucleare, Sezione Roma 1, Gruppo Sanità and Physics Laboratory, Istituto Superiore di Sanità, 00161 Roma, Italy

v TRIUMF, Vancouver, British Columbia V6T 2A3, Canada

${ }^{\mathrm{w}}$ Department of Physics, Tokyo Institute of Technology, Tokyo 152, Japan

${ }^{x}$ Department of Physics and Astronomy, Vrije Universiteit, 1081 HV Amsterdam, The Netherlands

${ }^{\mathrm{y}}$ Andrzej Soltan Institute for Nuclear Studies, 00-689 Warsaw, Poland

${ }^{\mathrm{z}}$ Yerevan Physics Institute, 375036 Yerevan, Armenia

\begin{abstract}
Azimuthal asymmetries in exclusive electroproduction of a real photon from an unpolarized deuterium target are measured with respect to beam helicity and charge. They appear in the distribution of these photons in the azimuthal angle $\phi$ around the virtual-photon direction, relative to the lepton scattering plane. The extracted asymmetries are attributed to either the deeply virtual Compton scattering process or its interference with the Bethe-Heitler process. They are compared with earlier results on the proton target. In the measured kinematic region, the beam-charge asymmetry amplitudes and the leading amplitudes of the beam-helicity asymmetries on an unpolarized deuteron target are compatible with the results from unpolarized protons.
\end{abstract}

Key words: DIS, HERMES experiment, GPD, DVCS, deuteron, unpolarized deuterium target PACS: 13.60.-r, 24.85.+p, 13.60.Fz, 14.20.Dh 


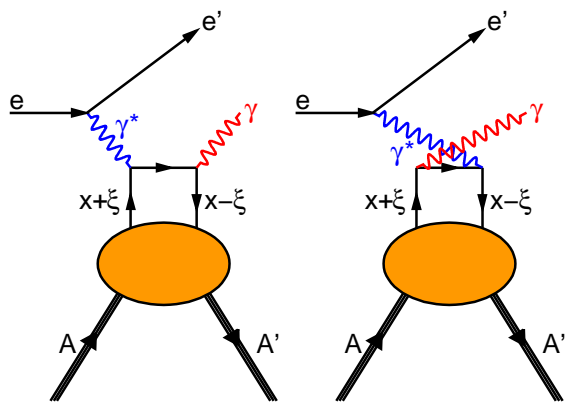

(a)

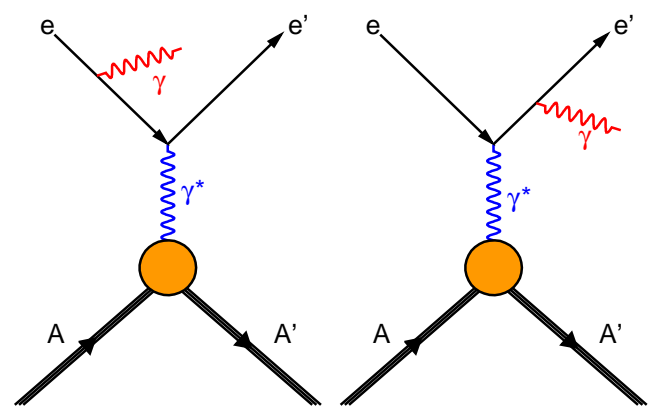

(b)

Fig. 1. Leading order Feynman diagrams for (a) deeply virtual Compton scattering and (b) the Bethe-Heitler process.

\section{Introduction}

Lepton-nucleon scattering experiments have long been an important tool in the detailed study of nucleon structure [1]. Two complementary approaches have contributed the most to our understanding of the nucleon. Elastic lepton-nucleon scattering has been exploited to extract nucleon form factors, which reveal how the electromagnetic nucleon structure differs from that of a point-like spin-1/2 particle. In another approach, Parton Distribution Functions (PDFs) are extracted from Deeply Inelastic Scattering (DIS). They represent distributions in the longitudinal momentum fraction carried by quarks and gluons in a nucleon moving with "infinite" momentum. PDFs and form factors present only one-dimensional pictures of nucleon structure. In recent years, a more comprehensive multi-dimensional description of the nucleon has emerged in the framework of Generalized Parton Distributions (GPDs) [2-4]. Their dependence on three kinematic

\footnotetext{
1 Now at: Old Dominion University, Norfolk, VA 23529, USA

2 Now at: Brookhaven National Laboratory, Upton, New York 11772-5000, USA

3 Now at: Massachusetts Institute of Technology, Cambridge, Massachusetts 02139, USA

4 Now at: Siemens AG Molecular Imaging, 91052 Erlangen, Germany

5 Now at: Institut für Physik, Universität Mainz, 55128 Mainz, Germany

6 Now at: IPN (UMR 8608) CNRS/IN2P3 - Universiteté Paris-Sud, 91406 Orsay, France

7 Now at: Institut für Kernphysik, Universität Frankfurt a.M., 60438 Frankfurt a.M., Germany

8 Now at: Siemens AG, 91301 Forchheim, Germany

9 Now at: Graduate University of Chinese Academy of Sciences, Beijing 100049, china

${ }^{10}$ Present address: Institut für Theoretische Physik II, Ruhr-Universität Bochum, 44780 Bochum, Germany

${ }^{11}$ Now at: Dept of Radiology, Stanford University, School of Medicine, Stanford, California 94305-5105, USA

12 Now at: Carnegie Mellon University, Pittsburgh, Pennsylvania 15213, USA

${ }^{13}$ Now at: IRCCS Multimedica Holding S.p.A., 20099 Sesto San Giovanni (MI), Italy

${ }^{14}$ Now at: AREVA NP GmbH, 91058 Erlangen, Germany

${ }^{15}$ Now at: Fermi National Accelerator Laboratory, Batavia, Illinois 60510, USA

${ }^{16}$ Now at: Thomas Jefferson National Accelerator Facility, Newport News, Virginia 23606, USA
} 


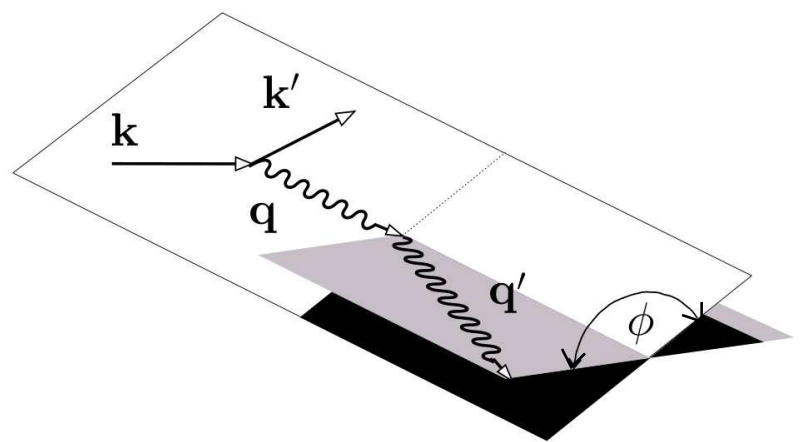

Fig. 2. Definition of the azimuthal angle $\phi$ between the lepton scattering and photon production planes. Note that the azimuthal angle defined in this work differs from that used in Ref. [15]: $\phi=\pi-\phi_{[15]}$.

quantities in addition to their evolution with the hard scale of the process carries information on two-parton correlations and quark transverse spatial distributions [5-10]. GPDs embody PDFs as limiting cases, while elastic form factors appear as certain GPD moments. Other moments are connected with the total parton angular momentum contribution to the nucleon spin via the Ji relation [4].

GPDs can be constrained by measurements of hard exclusive leptoproduction of a photon or meson in 'elastic' processes that leave the target intact. In Deeply Virtual Compton Scattering (DVCS), a quark absorbs a hard virtual photon, emits an energetic real photon and joins the target remnant (see Fig. 1 (a)). DVCS is presently the only experimentally feasible hard exclusive process for which the effects of next-to-leading order [11-13] and next-to-leading twist [14-16] are under complete theoretical control [17].

The final state of the DVCS process cannot be experimentally distinguished from that of the Bethe-Heitler (BH) process, i.e., radiative elastic scattering (see Fig. 1 (b)). Hence, the two processes can interfere. Exclusive leptoproduction on a nucleon or nuclear target $A$ of a real photon with four-momentum $q^{\prime}$ is denoted by

$$
e(k)+A(p) \rightarrow e\left(k^{\prime}\right)+A\left(p^{\prime}\right)+\gamma\left(q^{\prime}\right),
$$

where $k\left(k^{\prime}\right)$ and $p\left(p^{\prime}\right)$ are the four-momenta of the incoming (outgoing) lepton and target, respectively. Averaged over the kinematic acceptance of the HERMES experiment, the $\mathrm{BH}$ cross section is much larger than that of the DVCS process. However, the BH cross section has a much weaker $Q^{2}$ dependence than the evolution of the DVCS cross section [4], so that in the HERMES energy range they can become comparable near $Q^{2}=1 \mathrm{GeV}^{2}$, with $-Q^{2} \equiv q^{2}=\left(k-k^{\prime}\right)^{2}$.

Even in kinematic conditions where the DVCS process makes only a small contribution to the photon production cross section, its interference with the $\mathrm{BH}$ process provides access to the DVCS amplitudes through measurements of cross section asymmetries with respect to the charge and helicity of the incident lepton and the polarization of the target. These asymmetries appear in the distribution of the real photons in the azimuthal angle $\phi$, defined as the angle between the lepton scattering plane, i.e., the plane defined by the incoming and outgoing lepton direction and the photon production plane spanned by the virtual and real photons (see Fig. 2). Significant azimuthal beam-helicity asymmetries in hard electroproduction of photons on the proton were first reported in Refs. [18,19]. Later, asymmetries with respect to longitudinal [20,21] and transverse [22] target polarization, 
as well as beam charge [23] and, with greater precision, beam helicity [24-27], were also measured on the proton.

Measurements of azimuthal asymmetries for DVCS on nuclear targets [28] were advocated as a useful source of information about partonic behavior in nuclei and nuclear binding forces [29]. If the target nucleus remains in its ground state the process is called coherent, while it is called incoherent if the nucleus is broken up. The deuteron is a spin-1 nucleus, with implications for DVCS observables for the coherent reactions, which contribute mainly at very small values of the momentum transfer to the target. The asymmetries from the incoherent process involve mainly hard exclusive electroproduction of a photon on the proton. The neutron contribution to the yield is typically small due to the suppression of the $\mathrm{BH}$ amplitude on the neutron by the small elastic electric form factor at low and moderate values of the momentum transfer to the target.

This paper reports the first observation of azimuthal asymmetries with respect to beam helicity and charge for exclusive electroproduction of a real photon from an unpolarized deuterium target $\left(\boldsymbol{e}^{ \pm} d \rightarrow e^{ \pm} \gamma X\right)$. The dependence of these asymmetries on the kinematic conditions of the reaction is also presented and certain asymmetry amplitudes are compared with the corresponding amplitudes obtained on an unpolarized hydrogen target $\left(\boldsymbol{e}^{ \pm} p \rightarrow e^{ \pm} \gamma X\right)$ at HERMES [27].

\section{GPDs and DVCS}

\subsection{Generalized Parton Distributions}

In the generalized Bjorken limit of large $Q^{2}$ at fixed values of the Bjorken scaling variable $x_{B}=Q^{2} /(2 p \cdot q)$ and small squared four-momentum transfer $t=\left(p-p^{\prime}\right)^{2}$ to the target, the DVCS process can be described by the leading (handbag) diagrams in Fig. 1(a). Here, the process factorizes $[3,12,30]$ into a hard photon-quark scattering part calculable in quantum electrodynamics, and a soft part describing the nucleon structure, which can be expressed in terms of GPDs [2-4].

Like PDFs, GPDs depend on $x$ and on the factorization scale $Q^{2}$. In addition, GPDs depend on a skewness variable $\xi$ and the Mandelstam variable $t$. The skewness $\xi$ represents half the difference in the longitudinal momentum fractions of the quark before and after the scattering, while $x$ is their mean value (following the convention of Ref. [4]). In leading order, $\xi$ is directly accessible as it is related to the Bjorken scaling variable $x_{B}$ by $\xi \simeq x_{B} /\left(2-x_{B}\right)$. In contrast, $x$ is not directly accessible in DVCS, and some observables appear as $x$-convolutions of GPDs. Hence $x$ plays a role different from that of $x_{B}$ in inclusive DIS. GPDs evolve logarithmically with $Q^{2}$ in analogy with PDFs [2-4,31]. This dependence on $Q^{2}$ is omitted for simplicity in the following.

DVCS on spin-1/2 targets, such as nucleons, is described by four leading-twist quarkchirality conserving GPDs for each quark flavour $q$ (and also for the gluon $g$ ), namely the GPDs $H^{q}, E^{q}, \widetilde{H}^{q}$ and $\widetilde{E}^{q}[15]$. The GPDs $H^{q}$ and $E^{q}$ are quark-helicity averaged whereas $\widetilde{H}^{q}$ and $\widetilde{E}^{q}$ are quark-helicity dependent. The GPDs $H^{q}$ and $\widetilde{H}^{q}$ conserve nucleon-helicity while $E^{q}$ and $\widetilde{E}^{q}$ are associated with a helicity flip of the nucleon. In contrast, the coherent process on spin-1 nuclei, such as the deuteron, requires nine GPDs [32] $-H_{1}^{q}, H_{2}^{q}, H_{3}^{q}$, $H_{4}^{q}, H_{5}^{q}, \widetilde{H}_{1}^{q}, \widetilde{H}_{2}^{q}, \widetilde{H}_{3}^{q}$ and $\widetilde{H}_{4}^{q}$ - to describe all DVCS observables. In the forward limit of vanishing momentum difference between the initial and final hadronic state $(t \rightarrow 0$ and 
$\xi \rightarrow 0)$, the GPD $H^{q}(x, 0,0)$ reduces to $f_{1}^{q}(x)$, the quark number density distribution, and $\widetilde{H}^{q}(x, 0,0)$ reduces to $g_{1}^{q}(x)$, the quark helicity distribution. Similarly, for spin-1 targets the GPDs $H_{1}, \widetilde{H}_{1}$ and $H_{5}$ reduce to the following parton densities in the forward limit:

$$
\begin{aligned}
& H_{1}^{q}(x, 0,0)=\frac{q^{1}(x)+q^{-1}(x)+q^{0}(x)}{3} \equiv f_{1}^{q}(x), \\
& \widetilde{H}_{1}^{q}(x, 0,0)=q_{\rightarrow}^{1}(x)-q_{\rightarrow}^{-1}(x) \quad \equiv g_{1}^{q}(x), \\
& H_{5}^{q}(x, 0,0)=q^{0}(x)-\frac{q^{1}(x)+q^{-1}(x)}{2} \equiv b_{1}^{q}(x) \text {, }
\end{aligned}
$$

where $q_{\rightarrow[\leftarrow]}^{\Lambda}(x)$ represents the number density of a \{anti\} quark with momentum fraction $\{x<0\} x>0$ and positive [negative] helicity in a rapidly moving deuteron target with longitudinal spin projection $\Lambda$. The 'unpolarized' (polarization averaged) quark densities $q^{\Lambda}$ are defined as $q^{\Lambda}(x)=q_{\rightarrow}^{\Lambda}(x)+q_{\leftarrow}^{\Lambda}(x)$. While the probabilistic interpretation of polarization-averaged and polarization-difference structure functions $f_{1}(x)$ and $g_{1}(x)$ in terms of quark densities is similar to that in the spin- $1 / 2$ case, the tensor structure function $b_{1}(x)$ does not exist for spin- $1 / 2$ targets. It has been measured in DIS on a polarized spin-1 target [33]. Both $H_{3}$ and $H_{5}$ are associated with the $5 \% D$-wave component of the deuteron wave function in terms of nucleons [34]. $\mathrm{H}_{3}$ is related to isoscalar currents and probes the binding forces in the deuteron, and $H_{5}$ involves a tensor term [32,35], the analog of which has no relationship to any local current due to Lorentz invariance.

\subsection{Deeply virtual Compton scattering amplitudes}

For a target of atomic mass number $A$, the cross section for the hard exclusive leptoproduction of real photons is given by $[35,36]$

$$
\frac{\mathrm{d} \sigma}{\mathrm{d} x_{A} \mathrm{~d} Q^{2} \mathrm{~d}|t| \mathrm{d} \phi}=\frac{x_{A} e^{6}}{32(2 \pi)^{4} Q^{4}} \frac{|\mathcal{T}|^{2}}{\sqrt{1+\varepsilon^{2}}},
$$

where $x_{A} \equiv Q^{2} /\left(2 M_{A} \nu\right)$ is the nuclear Bjorken $x_{B}, M_{A}$ is the target mass and $\nu \equiv$ $p \cdot q / M_{A}, \varepsilon \equiv 2 x_{A} M_{A} / \sqrt{Q^{2}}$, and $\mathcal{T}$ is the total reaction amplitude.

As the final states of the DVCS and BH processes are indistinguishable, the cross section contains the square of the coherent sum of their amplitudes:

$$
|\mathcal{T}|^{2}=\left|\mathcal{T}_{\mathrm{BH}}+\mathcal{T}_{\mathrm{DVCS}}\right|^{2}=\left|\mathcal{T}_{\mathrm{BH}}\right|^{2}+\left|\mathcal{T}_{\mathrm{DVCS}}\right|^{2}+\underbrace{\mathcal{T}_{\mathrm{DVCS}} \mathcal{T}_{\mathrm{BH}}^{*}+\mathcal{T}_{\mathrm{DVCS}}^{*} \mathcal{T}_{\mathrm{BH}}}_{\mathrm{I}} .
$$

Here, I denotes the BH-DVCS interference term. The BH amplitude is calculable to leading order in Quantum Electrodynamics (QED) using nuclear form factors measured in elastic scattering.

The interference term I in Eq. 6 provides separate experimental access to the real and imaginary parts of the DVCS amplitude through measurements of various cross-section asymmetries as functions of the azimuthal angle $\phi$ [36]. Each of the three terms of Eq. 6 can be written as a Fourier series in $\phi[15]$, which in the case of an unpolarized target reads 


$$
\begin{aligned}
& \left|\mathcal{T}_{\mathrm{BH}}\right|^{2}=\frac{K_{\mathrm{BH}}}{\mathcal{P}_{1}(\phi) \mathcal{P}_{2}(\phi)} \times \sum_{n=0}^{2} c_{n}^{\mathrm{BH}} \cos (n \phi), \\
& \left|\mathcal{T}_{\mathrm{DVCS}}\right|^{2}=K_{\mathrm{DVCS}} \times\left\{c_{0}^{\mathrm{DVCS}}+\sum_{n=1}^{2} c_{n}^{\mathrm{DVCS}} \cos (n \phi)+\lambda s_{1}^{\mathrm{DVCS}} \sin \phi\right\}, \\
& \mathrm{I}=-\frac{K_{\mathrm{I}} e_{\ell}}{\mathcal{P}_{1}(\phi) \mathcal{P}_{2}(\phi)} \times\left\{c_{0}^{\mathrm{I}}+\sum_{n=1}^{3} c_{n}^{\mathrm{I}} \cos (n \phi)+\lambda \sum_{n=1}^{2} s_{n}^{\mathrm{I}} \sin (n \phi)\right\} .
\end{aligned}
$$

Here, $K_{\mathrm{BH}}, K_{\mathrm{DVCS}}$, and $K_{\mathrm{I}}$ are kinematic factors, $e_{\ell}$ denotes the lepton beam charge in units of the elementary charge, and $\lambda$ the helicity of the longitudinally polarized lepton beam. The squared $\mathrm{BH}$ and interference terms have an additional $\cos \phi$ dependence in the denominator due to the lepton propagators $\mathcal{P}_{1}(\phi)$ and $\mathcal{P}_{2}(\phi)$ in the $\mathrm{BH}$ process $[15,36]$.

The Fourier coefficients $c_{n}^{\mathrm{I}}$ and $s_{n}^{\mathrm{I}}$ in Eq. 9 can be expressed as linear combinations of Compton Form Factors $\mathcal{F}(\xi, t)$ (CFFs) [35], which in turn are convolutions of the corresponding $\operatorname{GPDs} F^{q}(x, \xi, t)$ with the hard scattering coefficient functions $\mathcal{C}_{q}^{\mp}[11-13]$ :

$$
\mathcal{F}(\xi, t)=\sum_{q} \int_{-1}^{1} \mathrm{~d} x \mathcal{C}_{q}^{\mp}(\xi, x) F^{q}(x, \xi, t),
$$

where the $-\{+\}$ sign applies to $F^{q}=H_{1}^{q}, \ldots, H_{5}^{q}\left\{\widetilde{H}_{1}^{q}, \ldots, \widetilde{H}_{4}^{q}\right\}$ in the case of a spin-1 target. The real and imaginary parts of the CFFs have different relationships to the flavor sum over the respective quark GPDs. To leading order in $\alpha_{s}$,

$$
\Im \mathrm{m}\{\mathcal{F}(\xi, t)\}=-\pi \sum_{q} e_{q}^{2}\left(F^{q}(\xi, \xi, t) \mp F^{q}(-\xi, \xi, t)\right) .
$$

Hence measurements of cross-section asymmetries with respect to the beam helicity directly determine combinations of GPDs along the lines $x= \pm \xi$. In contrast, the real parts of the CFFs involve the full interval in $x$ and constrain the $x$ dependence of GPDs through convolutions:

$$
\Re \mathrm{e}\{\mathcal{F}(\xi, t)\}=\sum_{q} e_{q}^{2}\left[P \int_{-1}^{1} \mathrm{~d} x F^{q}(x, \xi, t)\left(\frac{1}{x-\xi} \pm \frac{1}{x+\xi}\right)\right],
$$

to leading order in $\alpha_{s}$. Here, $P$ denotes Cauchy's principal value. Since the $x$ dependence of GPDs is thereby only weakly constrained, experimental asymmetries in beam charge must be compared to the predictions of various GPD models.

At leading twist (twist-2), the coefficients $c_{1}^{\mathrm{I}}$ and $s_{1}^{\mathrm{I}}$ are related to the same combination of GPDs. This is also true for the kinematically suppressed coefficient $c_{0}^{\mathrm{I}} \propto-\frac{\sqrt{-t}}{Q} c_{1}^{\mathrm{I}}$. The coefficients $c_{0}^{\mathrm{I}}$ and $c_{1}^{\mathrm{I}}$ are sensitive to the 'D-term' [37,38], which contributes only in the 'ERBL' region $-\xi<x<\xi$ where quark GPDs have the characteristics of distribution amplitudes for the creation of a quark-antiquark pair. It does not contribute in the complementary 'DGLAP' region $|x|>\xi$, where quark GPDs describe the emission and reabsorption of an (anti-)quark in the infinite momentum frame, thereby having properties analogous to the familiar (anti-)quark distribution functions. The D-term provides a convenient means of representing this profound difference in GPD properties between the two regions, while, e.g., the absorption of this contribution into the double distributions $[2,3]$ would require the introduction of terms with unnatural divergence, having 
a severity beyond representation by delta functions or their derivatives. In addition to $c_{1}^{\mathrm{I}}, s_{1}^{\mathrm{I}}$, and $c_{0}^{\mathrm{I}}$, the only other Fourier coefficient related to only twist-2 quark GPDs is $c_{0}^{\text {DVCS }}$. The coefficients $c_{1}^{\text {DVCS }}, s_{1}^{\text {DVCS }}, c_{2}^{\mathrm{I}}$, and $s_{2}^{\mathrm{I}}$ appear at the twist-3 level, while $c_{2}^{\text {DVCS }}$ and $c_{3}^{\mathrm{I}}$ arise from the gluonic transversity operator [39-41] at twist-2 level. The highest harmonics of the interference and squared DVCS terms may also receive a twist-4 contribution [42].

\subsection{Azimuthal cross section asymmetries}

The beam-helicity asymmetries for a longitudinally (L) polarized lepton beam and an unpolarized (U) target, based on the difference and sum of yields for the two beam charges, respectively, are defined as

$$
\begin{gathered}
\mathcal{A}_{\mathrm{LU}}^{\mathrm{I}}(\phi) \equiv \frac{\left[\mathrm{d} \sigma^{+\rightarrow}(\phi)-\mathrm{d} \sigma^{+\leftarrow}(\phi)\right]-\left[\mathrm{d} \sigma^{-\rightarrow}(\phi)-\mathrm{d} \sigma^{-\leftarrow}(\phi)\right]}{\left[\mathrm{d} \sigma^{+\rightarrow}(\phi)+\mathrm{d} \sigma^{+\leftarrow}(\phi)\right]+\left[\mathrm{d} \sigma^{-\rightarrow}(\phi)+\mathrm{d} \sigma^{-\leftarrow}(\phi)\right]}, \\
\mathcal{A}_{\mathrm{LU}}^{\mathrm{DVCS}}(\phi) \equiv \frac{\left[\mathrm{d} \sigma^{+\rightarrow}(\phi)-\mathrm{d} \sigma^{+\leftarrow}(\phi)\right]+\left[\mathrm{d} \sigma^{-\rightarrow}(\phi)-\mathrm{d} \sigma^{-\leftarrow}(\phi)\right]}{\left[\mathrm{d} \sigma^{+\rightarrow}(\phi)+\mathrm{d} \sigma^{+\leftarrow}(\phi)\right]+\left[\mathrm{d} \sigma^{-\rightarrow}(\phi)+\mathrm{d} \sigma^{-\leftarrow}(\phi)\right]},
\end{gathered}
$$

where $\rightarrow(\leftarrow)$ denotes positive (negative) beam helicity and the superscript $+(-)$ corresponds to positron (electron) beam. These definitions serve to separate the $\sin (n \phi)$ terms in Eqs. 8 and 9. Similarly, the beam-charge asymmetry (BCA) for an unpolarized beam scattering from this target is defined as

$$
\begin{aligned}
\mathcal{A}_{\mathrm{C}}(\phi) & \equiv \frac{\mathrm{d} \sigma^{+}(\phi)-\mathrm{d} \sigma^{-}(\phi)}{\mathrm{d} \sigma^{+}(\phi)+\mathrm{d} \sigma^{-}(\phi)} \\
& =\frac{\left[\mathrm{d} \sigma^{+\rightarrow}(\phi)+\mathrm{d} \sigma^{+\leftarrow}(\phi)\right]-\left[\mathrm{d} \sigma^{-\rightarrow}(\phi)+\mathrm{d} \sigma^{-\leftarrow}(\phi)\right]}{\left[\mathrm{d} \sigma^{+\rightarrow}(\phi)+\mathrm{d} \sigma^{+\leftarrow}(\phi)\right]+\left[\mathrm{d} \sigma^{-\rightarrow}(\phi)+\mathrm{d} \sigma^{-\leftarrow}(\phi)\right]} .
\end{aligned}
$$

In terms of the Fourier coefficients of Eqs. 7-9 these equations read as

$$
\begin{aligned}
\mathcal{A}_{\mathrm{LU}}^{\mathrm{I}}(\phi) & =\frac{-\frac{K_{\mathrm{I}}}{\mathcal{P}_{1}(\phi) \mathcal{P}_{2}(\phi)} \sum_{n=1}^{2} s_{n}^{\mathrm{I}} \sin (n \phi)}{\frac{K_{\mathrm{BH}}}{\mathcal{P}_{1}(\phi) \mathcal{P}_{2}(\phi)} \sum_{n=0}^{2} c_{n}^{\mathrm{BH}} \cos (n \phi)+K_{\mathrm{DVCS}} \sum_{n=0}^{2} c_{n}^{\mathrm{DVCS}} \cos (n \phi)}, \\
\mathcal{A}_{\mathrm{LU}}^{\mathrm{DVCS}}(\phi) & =\frac{K_{\mathrm{DVCS}} s_{1}^{\mathrm{DVCS}} \sin \phi}{\frac{K_{\mathrm{BH}}}{\mathcal{P}_{1}(\phi) \mathcal{P}_{2}(\phi)} \sum_{n=0}^{2} c_{n}^{\mathrm{BH}} \cos (n \phi)+K_{\mathrm{DVCS}} \sum_{n=0}^{2} c_{n}^{\mathrm{DVCS}} \cos (n \phi)}, \\
\mathcal{A}_{\mathrm{C}}(\phi) & =\frac{-\frac{K_{\mathrm{I}}}{\mathcal{P}_{1}(\phi) \mathcal{P}_{2}(\phi)} \sum_{n=0}^{3} c_{n}^{\mathrm{I}} \cos (n \phi)}{\frac{K_{\mathrm{BH}}}{\mathcal{P}_{1}(\phi) \mathcal{P}_{2}(\phi)} \sum_{n=0}^{2} c_{n}^{\mathrm{BH}} \cos (n \phi)+K_{\mathrm{DVCS}} \sum_{n=0}^{2} c_{n}^{\mathrm{DVCS}} \cos (n \phi)} .
\end{aligned}
$$

At leading twist (twist-2, twist-3, and twist-2, respectively in the preceding three equations), and neglecting gluonic terms, they reduce to

$$
\begin{gathered}
\mathcal{A}_{\mathrm{LU}}^{\mathrm{I}}(\phi) \simeq \frac{-\frac{K_{\mathrm{I}}}{\mathcal{P}_{1}(\phi) \mathcal{P}_{2}(\phi)} s_{1}^{\mathrm{I}} \sin \phi}{\frac{K_{\mathrm{BH}}}{\mathcal{P}_{1}(\phi) \mathcal{P}_{2}(\phi)} \sum_{n=0}^{2} c_{n}^{\mathrm{BH}} \cos (n \phi)+K_{\mathrm{DVCS}} c_{0}^{\mathrm{DVCS}}}, \\
\mathcal{A}_{\mathrm{LU}}^{\mathrm{DVCS}}(\phi) \simeq \frac{K_{\mathrm{DVCS}} s_{1}^{\mathrm{DVCS}} \sin \phi}{\frac{K_{\mathrm{BH}}}{\mathcal{P}_{1}(\phi) \mathcal{P}_{2}(\phi)} \sum_{n=0}^{2} c_{n}^{\mathrm{BH}} \cos (n \phi)+K_{\mathrm{DVCS}} c_{0}^{\mathrm{DVCS}}}, \\
\mathcal{A}_{\mathrm{C}}(\phi) \simeq \frac{-\frac{K_{\mathrm{I}}}{\mathcal{P}_{1}(\phi) \mathcal{P}_{2}(\phi)}\left(c_{0}^{\mathrm{I}}+c_{1}^{\mathrm{I}} \cos \phi\right)}{\frac{K_{\mathrm{BH}}}{\mathcal{P}_{1}(\phi) \mathcal{P}_{2}(\phi)} \sum_{n=0}^{2} c_{n}^{\mathrm{BH}} \cos (n \phi)+K_{\mathrm{DVCS}} c_{0}^{\mathrm{DVCS}}} .
\end{gathered}
$$


To the extent that the DVCS contributions to the common denominator can be neglected at HERMES kinematics, the lepton propagators $\mathcal{P}_{1}(\phi)$ and $\mathcal{P}_{2}(\phi)$ cancel in Eqs. 16, 18, 19, and 21. However, this approximation is not invoked in the following because it would be subject to substantial model uncertainty.

\subsection{From Compton form factors to asymmetries}

Measured asymmetries are used to constrain GPD models by direct comparison of the data with model predictions. However, it is instructive to consider certain approximations relating CFFs and thereby GPDs to observed asymmetries. (These approximations are not needed in the comparison of GPD model predictions with measured asymmetries.)

For an unpolarized nucleon target, the photon-helicity-conserving amplitude $\widetilde{\mathcal{M}}^{1,1}$ is given at leading twist by a linear combination of the CFFs $\mathcal{H}, \widetilde{\mathcal{H}}$ and $\mathcal{E}$, together with the Dirac and Pauli form factors $F_{1}$ and $F_{2}[15]$ :

$$
\widetilde{\mathcal{M}}^{1,1}=F_{1} \mathcal{H}+\frac{x_{N}}{2-x_{N}}\left(F_{1}+F_{2}\right) \widetilde{\mathcal{H}}-\frac{t}{4 M_{N}^{2}} F_{2} \mathcal{E},
$$

where $x_{N}$ is the Bjorken variable for the nucleon and $M_{N}$ is the nucleon mass. At small values of $x_{N}$ and $-t, \widetilde{\mathcal{M}}^{1,1} \simeq F_{1} \mathcal{H}$ for the proton. For the neutron, the term containing the $\mathrm{CFF} \mathcal{E}$ in Eq. 22 becomes substantial at large $-t$ due to the relative magnitudes of the form factors $F_{1}$ and $F_{2}$ for the neutron. The leading Fourier coefficients of the interference term can be approximated as $s_{1}^{\mathrm{I}} \propto \Im \mathrm{m} \widetilde{\mathcal{M}}^{1,1}$ and $c_{1}^{\mathrm{I}} \propto \Re \mathrm{e} \widetilde{\mathcal{M}}^{1,1}$. To leading order in 1/Q and in HERMES kinematic conditions,

$$
\begin{aligned}
\mathcal{A}_{\mathrm{LU}}^{\mathrm{I}}(\phi) & \propto-\frac{\Im \mathrm{m} \mathcal{H}}{F_{1}} \sin \phi, \\
\mathcal{A}_{\mathrm{C}}(\phi) & \propto \frac{\Re \mathrm{e} \mathcal{H}}{F_{1}} \cos \phi .
\end{aligned}
$$

For the coherent process on the deuteron, the relationship between the Fourier coefficients and the GPDs is complicated. However, the coefficients can be expanded in powers of $x_{D}$, the Bjorken variable for the deuteron target, and $\tau=t /\left(4 M_{D}^{2}\right)$, where $M_{D}$ is the deuteron mass [35]. Then, to leading order in $\alpha_{s}$ and $1 / Q, \mathcal{A}_{\mathrm{LU}}^{\mathrm{I}}(\phi)$ can be expressed in terms of the imaginary part of the deuteron CFFs $\mathcal{H}_{1}, \mathcal{H}_{3}$ and $\mathcal{H}_{5}$ and the deuteron elastic form factors [43] $G_{1}$ and $G_{3}$ (see Fig. 3). The quantity $|\tau|$ is typically about 0.003 in the range of small $-t$ where the coherent process is significant, extending up to values of $\tau$ only as large as 0.01 . However, as shown in Fig. 3, the magnitude of $G_{3}$ exceeds that of $G_{1}$ by more than one order of magnitude. Hence certain terms leading in $\tau$ (but not $\left.x_{D}\right)$ are retained. Defining

$$
\widetilde{\mathcal{D}}_{\mathrm{U}}^{1,1} \equiv \frac{3 G_{1} \mathcal{H}_{1}-2 \tau\left[G_{1} \mathcal{H}_{3}+G_{3}\left(\mathcal{H}_{1}-\frac{1}{3} \mathcal{H}_{5}\right)\right]+4 \tau^{2} G_{3} \mathcal{H}_{3}}{3 G_{1}^{2}-4 \tau G_{1} G_{3}+4 \tau^{2} G_{3}^{2}},
$$

the kinematic expansion yields

$$
\mathcal{A}_{\mathrm{LU}}^{\mathrm{I}}(\phi) \simeq-\frac{x_{D}(2-y) \sqrt{\frac{-t}{Q^{2}}(1-y)}}{2-2 y+y^{2}} \Im \mathrm{m} \widetilde{\mathcal{D}}_{\mathrm{U}}^{1,1} \sin \phi,
$$

where $y \equiv p \cdot q /(p \cdot k)$. 


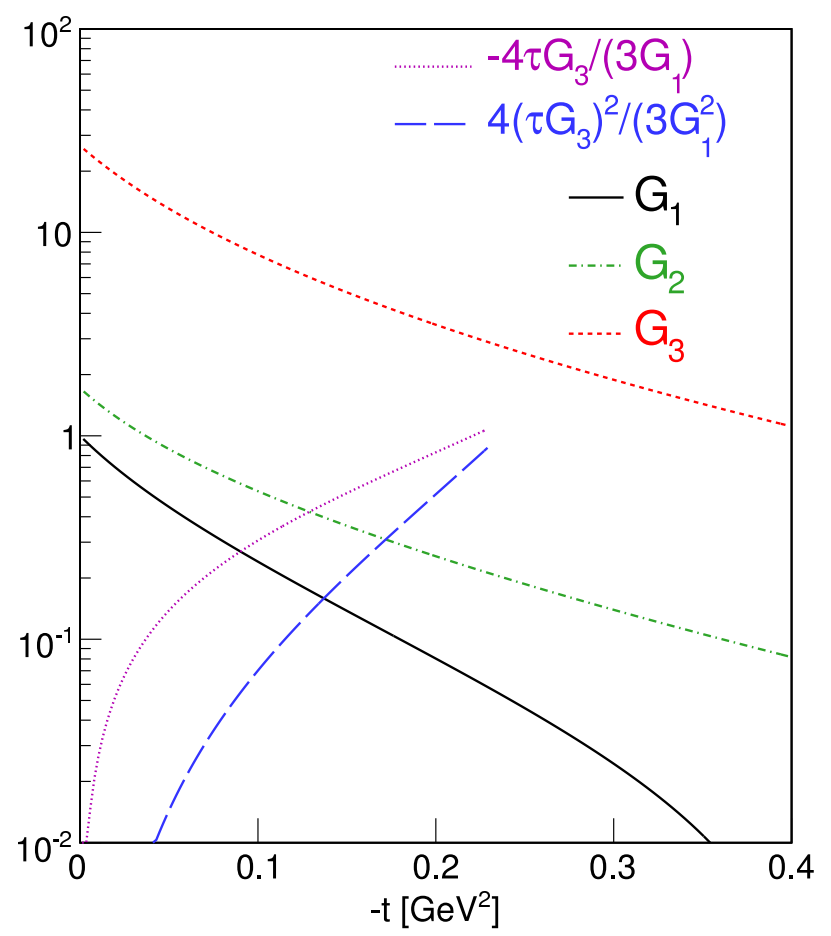

Fig. 3. The deuteron elastic form factors according to Parameterization II of Ref. [43], and the relative contributions to the denominator of, e.g., Eq. 25 of certain terms involving $G_{3}$ that are not leading in $\tau$.

The relative contributions of those terms are also shown in Fig. 3; they are less than $10 \%$ at $-t<0.03 \mathrm{GeV}^{2}$. When these terms are neglected, Eq. 26 becomes

$$
\mathcal{A}_{\mathrm{LU}}^{\mathrm{I}}(\phi) \simeq-\frac{x_{D}(2-y) \sqrt{\frac{-t}{Q^{2}}(1-y)}}{2-2 y+y^{2}} \frac{\Im \mathrm{m} \mathcal{H}_{1}}{G_{1}} \sin \phi .
$$

The deuteron $\mathcal{A}_{\mathrm{C}}(\phi)$ is related to the real part of the same linear combination of CFFs appearing in the deuteron $\mathcal{A}_{\mathrm{LU}}^{\mathrm{I}}(\phi)$ :

$$
\begin{array}{r}
\mathcal{A}_{\mathrm{C}}(\phi) \simeq-\frac{x_{D} \sqrt{\frac{-t}{Q^{2}}(1-y)}}{y} \Re \mathrm{e} \widetilde{\mathcal{D}}_{\mathrm{U}}^{1,1} \cos \phi \\
\simeq-\frac{x_{D} \sqrt{\frac{-t}{Q^{2}}(1-y)}}{y} \frac{\Re \mathrm{e} \mathcal{H}_{1}}{G_{1}} \cos \phi .
\end{array}
$$

For the coherent process on the deuteron, the leading term in the expansion of coefficients $s_{1}^{\mathrm{I}}$ and $c_{1}^{\mathrm{I}}$ lead respectively to Eqs. 27 and 29 which are analogous to Eqs. 23 and 24 for scattering on the nucleon. 
Table 1

The beam charge and polarization as well as the integrated luminosity in $\mathrm{pb}^{-1}$ of the data sets used for the extraction of the various asymmetries on the unpolarized deuterium target.

\begin{tabular}{|c|c|c|c|c|c|}
\hline \multirow{3}{*}{ Year } & \multirow{3}{*}{$\begin{array}{l}\text { Beam } \\
\text { Charge }\end{array}$} & \multicolumn{2}{|c|}{ Beam } & \multicolumn{2}{|c|}{ Luminosity } \\
\hline & & \multicolumn{2}{|c|}{ Polarization } & \multicolumn{2}{|c|}{$\left[\mathrm{pb}^{-1}\right]$} \\
\hline & & $\lambda=-1$ & $=+1$ & $\lambda=-1$ & $=+1$ \\
\hline 1996 & $e^{+}$ & & 0.516 & & 43.9 \\
\hline 1997 & $e^{+}$ & -0.511 & & 53.1 & \\
\hline 1998 & $e^{-}$ & -0.307 & & 24.1 & \\
\hline 1999 & $e^{+}$ & -0.552 & 0.418 & 0.9 & 5.1 \\
\hline 2000 & $e^{+}$ & -0.584 & 0.552 & 29.7 & 9.0 \\
\hline 2005 & $e^{-}$ & -0.355 & 0.377 & 66.3 & 65.7 \\
\hline Sum & & & & 174.1 & 123.7 \\
\hline
\end{tabular}

\section{The HERMES experiment}

A detailed description of the HERMES experiment can be found in Ref. [44]. A longitudinally polarized positron or electron beam of $27.6 \mathrm{GeV}$ energy was scattered from an unpolarized deuterium gas target internal to the HERA lepton storage ring at DESY. The lepton beam was transversely polarized via the asymmetry in the emission of synchrotron radiation (Sokolov-Ternov effect) [45] in the arcs of the HERA storage ring. The transverse beam polarization was transformed locally into longitudinal polarization by a pair of spin rotators located before and after the experiment [46]. The helicity of the beam was typically reversed approximately every two months.

The beam polarization was continuously monitored by two Compton backscattering polarimeters $[47,48]$. The average values of the beam polarization for various running periods are given in Table 1; the average fractional systematic uncertainty was $2.4 \%$. The scattered leptons and produced particles were detected in the polar angle range $0.04 \mathrm{rad}<\theta<0.22 \mathrm{rad}$. The lepton trigger required a coincidence of signals from scintillator hodoscope planes and the local deposition of a minimum energy of $3.5 \mathrm{GeV}$ in the electromagnetic calorimeter. Lepton identification was accomplished using the transition-radiation detector, the preshower scintillator counter, and the electromagnetic calorimeter. The average lepton identification efficiency was at least $98 \%$ with hadron contamination that was less than $1 \%$. Photons were identified by the detection of energy deposited in the calorimeter and preshower counter with no associated charged-particle track. Data quality requirements were applied to the event sample to ensure the uniform response of the spectrometer.

\section{Event selection and yield distributions}

The data sets used in the extraction of the various asymmetries reported here are given in Table 1. In this analysis, it was required that events contained exactly one chargedparticle track consistent with being the scattered beam lepton, and a single cluster in the calorimeter with an energy deposit $E_{\gamma}>5.0 \mathrm{GeV}$ and with no associated charged 


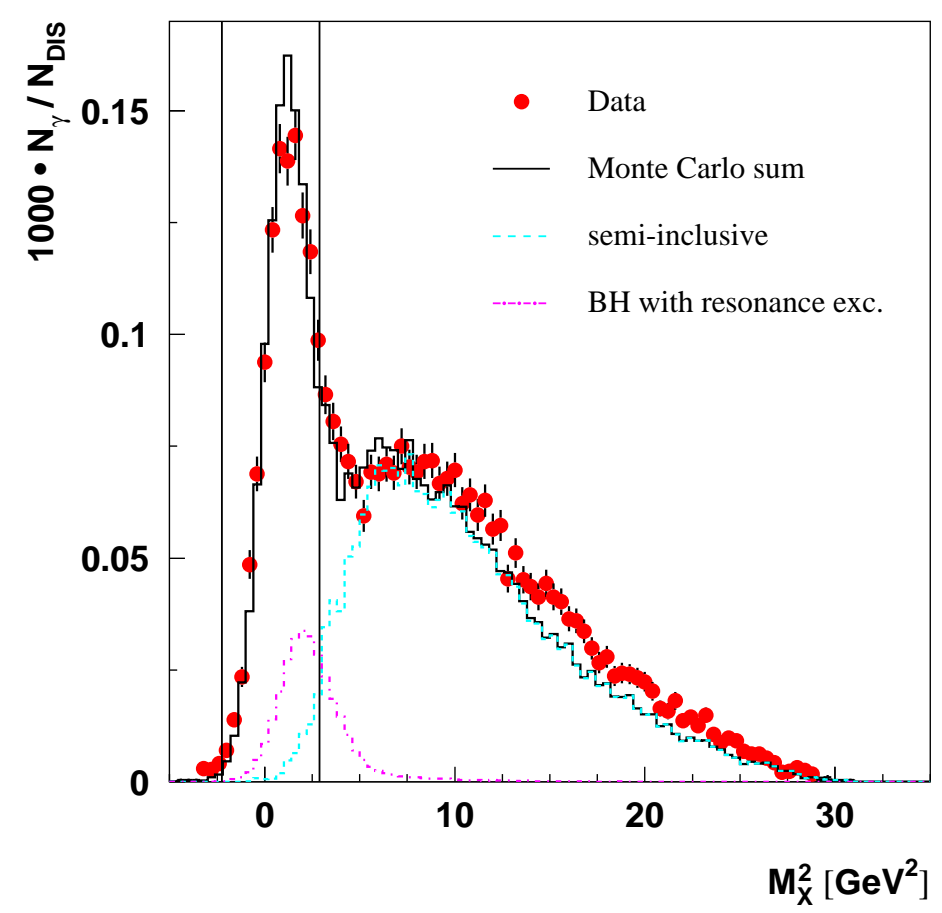

Fig. 4. The measured distribution (points) of electroproduced real-photon events versus the squared missing mass $M_{X}^{2}$. The solid curve represents a Monte Carlo simulation including coherent and incoherent $\mathrm{BH}$ and DVCS processes, the BH processes with the excitation of resonant final states (represented separately by the dashed-dotted curve), and the semi-inclusive background (dashed curve). The simulations and data are both normalized to the number of DIS events. The region between the two vertical lines indicates the selected exclusive events. Data from the year 2005 are shown.

track. The following requirements were imposed on the event kinematics: $1 \mathrm{GeV}^{2}<$ $Q^{2}<10 \mathrm{GeV}^{2}, W_{N}^{2}>9 \mathrm{GeV}^{2}, \nu<22 \mathrm{GeV}$ and $0.03<x_{N}<0.35$, where $W_{N}^{2}=$ $M_{N}^{2}+2 M_{N} \nu-Q^{2}, x_{N}=Q^{2} /\left(2 M_{N} \nu\right)$, and $\nu \equiv p \cdot q / M_{N}$. The nucleonic (proton) mass $M_{N}$ was used in all kinematic constraints on event selection even at small values of $-t$, where coherent reactions on the deuteron are dominant, because the experiment did not distinguish between coherent and incoherent scattering and the latter dominates over most of the kinematic range. Monte Carlo studies have shown that this choice has little effect on the extracted asymmetries [49]. In order to reduce background from the decay of neutral mesons, the angle between the laboratory 3-momenta of the real and virtual photons was limited to $\theta_{\gamma^{*} \gamma}<45 \mathrm{mrad}$. The minimum angle requirement $\theta_{\gamma^{*} \gamma}>$ 5 mrad was chosen according to Monte Carlo studies to be compatible with the effects of instrumental resolution in the determination of $\phi$.

'Exclusive' single-photon events were selected by requiring the squared missing mass $M_{X}^{2}$ to be close to the squared nucleon mass $M_{N}^{2}$, where $M_{X}^{2}$ is defined as $M_{X}^{2}=$ $\left(q+P_{N}-q^{\prime}\right)^{2}$ with $P_{N}=\left(M_{N}, 0,0,0\right)$. Due to the finite resolution of the spectrometer and the calorimeter, $M_{X}^{2}$ may be negative. In Fig. 4 , the squared missing mass distribution of the selected events is compared with the predictions of Monte Carlo simulations of processes that contribute to both signal and background. One of the simulations uses an exclusive-photon generator for the BH and DVCS processes, including coherent and 


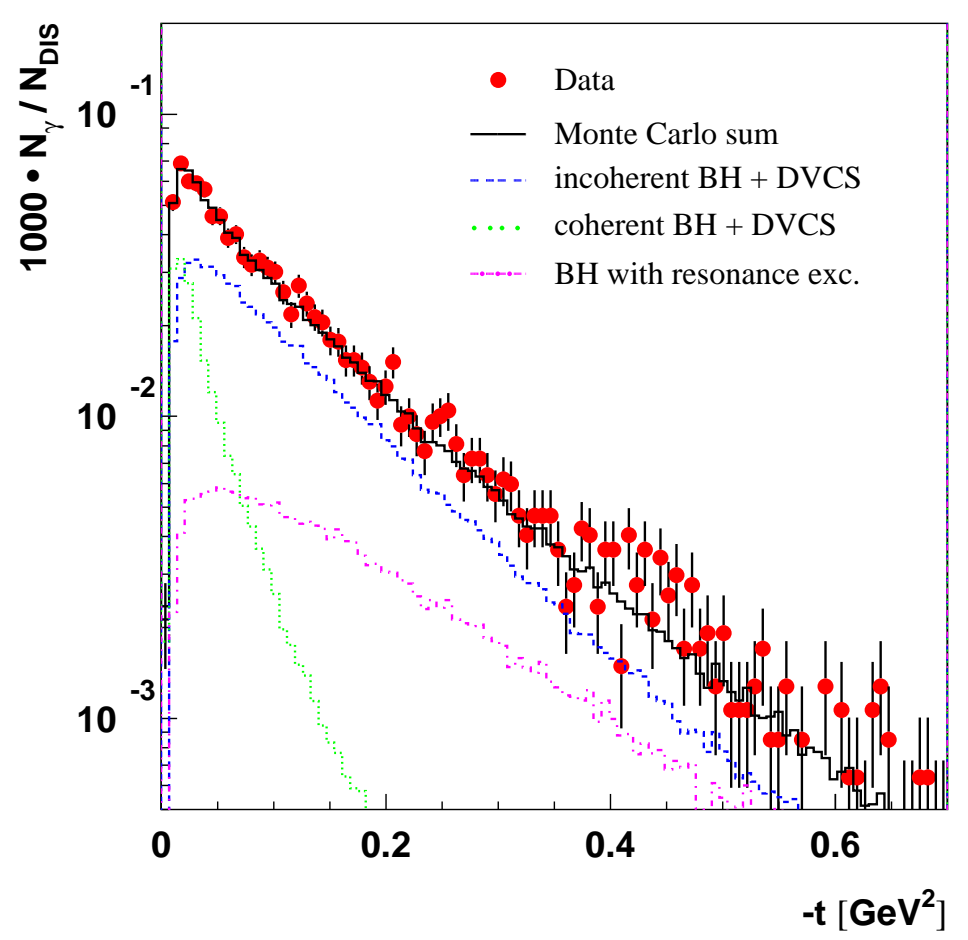

Fig. 5. Distribution in $-t$ of events selected in the exclusive region of $M_{X}^{2}$. The points represent experimental data while the continuous curve represents the simulation of real-photon production for all exclusive final states including resonances. Background from $\pi^{0}$ decay is not included. The dotted and dashed curves represent the BH plus DVCS contributions of the coherent and incoherent elastic process, respectively. The dash-dotted curve shows the resonant $\mathrm{BH}$ contributions. The simulations and data are both normalized to the number of DIS events. Data from the year 2005 are shown.

incoherent reactions as well as the excitation of resonant final states (a category known as associated production). The DVCS simulation for incoherent reactions on the nucleon is based on Ref. [50], while that for coherent reactions on the deuteron is based on the model from Ref. [35]. Most of the background in the vicinity of the exclusive peak comes from the decay of neutral pions. The dominant source of neutral pions is semi-inclusive DIS, $\gamma^{*} N \rightarrow \pi^{0} X \rightarrow \gamma \gamma X$, which is simulated using the LEPTO event generator [51] with a set of JETSET [52] fragmentation parameters tuned for HERMES kinematic conditions [53]. In this simulation, the photon originates mainly from decay of $\pi^{0} \mathrm{~s}$ from DIS fragmentation. Incoherent exclusive $\pi^{0}$ production, $\gamma^{*} N \rightarrow \pi^{0} N$, was simulated using an exclusive Monte Carlo event generator based on the GPD models of Ref. [54] and was found to be negligible $[49,55]$. HERMES data support this estimate [56]. The Monte Carlo yield exceeds the data by approximately $2 \%$ in the exclusive region. This may be due to the contribution of the DVCS process in the simulation of both coherent and incoherent processes, which is highly model-dependent and can vary between $10 \%$ and $25 \%$ [55] for the incoherent processes. On the other hand, radiative effects not included in the simulation would move events from the peak to the continuum [57].

Events were selected in the 'exclusive region', defined as $-(1.5)^{2} \mathrm{GeV}^{2}<M_{X}^{2}<$ $(1.7)^{2} \mathrm{GeV}^{2}$ to minimize background from DIS fragmentation while maintaining rea- 
sonable efficiency [58].

As the recoiling target nucleon or nucleus was undetected, the Mandelstam variable $t$ must be reconstructed from the measured kinematics of the scattered lepton and the detected photon. The resolution in the photon energy from the calorimeter is inadequate for a precise determination of $t$. Hence for events selected in the exclusive region in $M_{X}^{2}$, the final state is assumed to be exclusive, leaving the target intact, thereby allowing $t$ to be reconstructed with improved resolution using only the photon direction and the lepton kinematics [23]:

$$
t=\frac{-Q^{2}-2 \nu\left(\nu-\sqrt{\nu^{2}+Q^{2}} \cos \theta_{\gamma^{*} \gamma}\right)}{1+\frac{1}{M_{N}}\left(\nu-\sqrt{\nu^{2}+Q^{2}} \cos \theta_{\gamma^{*} \gamma}\right)} .
$$

The further restriction $-t<0.7 \mathrm{GeV}^{2}$ is imposed in the selection of exclusive events in order to reduce background from the decay of neutral mesons.

The $t$ distribution of events for the deuterium target is shown in Fig. 5 and compared with the Monte Carlo simulations discussed above. The simulated contributions of coherent and incoherent processes on the deuteron are also shown separately. Coherent scattering on the deuteron occurs preferentially at small values of $-t$. The Monte Carlo simulation shows that requiring $-t<0.06 \mathrm{GeV}^{2}$ enhances the mean fractional contribution of the coherent process from $20 \%$ to $40 \%$ in the HERMES spectrometer acceptance. Requiring $-t<0.01 \mathrm{GeV}^{2}$ can further enhance the coherent contribution to $66 \%$, but only at the cost of a rapidly decreasing yield. In Sections 6.1 and 6.2 , the first two $-t$ bins covering the range $0.00-0.06 \mathrm{GeV}^{2}$ will provide a measure of coherent effects; in Section 6.3 , an attempt is made to isolate the coherent contribution.

\section{Analysis of the data}

\subsection{Extraction of azimuthal asymmetry amplitudes}

The distribution of the expectation value of the yield for scattering a polarized lepton beam from an unpolarized deuterium target is given by

$$
\begin{aligned}
\langle\mathcal{N}\rangle\left(P_{\ell}, e_{\ell}, \phi\right) & =\mathcal{L}\left(P_{\ell}, e_{\ell}\right) \eta\left(e_{\ell}, \phi\right) \sigma_{\mathrm{UU}}(\phi) \\
& \times\left[1+P_{\ell} \mathcal{A}_{\mathrm{LU}}^{\mathrm{DVCS}}(\phi)+e_{\ell} \mathcal{A}_{\mathrm{C}}(\phi)+e_{\ell} P_{\ell} \mathcal{A}_{\mathrm{LU}}^{\mathrm{I}}(\phi)\right] .
\end{aligned}
$$

Here, $\mathcal{L}$ denotes the integrated luminosity, $P_{\ell}$ the longitudinal beam polarization, $\eta$ the detection efficiency, and $\sigma_{\mathrm{UU}}(\phi)$ the cross section for an unpolarized target averaged over both beam charges and both beam helicities, which can be expressed as

$$
\begin{aligned}
& \sigma_{\mathrm{UU}}(\phi)=\frac{x_{D}}{32(2 \pi)^{4} Q^{4}} \frac{1}{\sqrt{1+\varepsilon^{2}}} \\
& \times\left\{\frac{K_{\mathrm{BH}}}{\mathcal{P}_{1}(\phi) \mathcal{P}_{2}(\phi)} \sum_{n=0}^{2} c_{n}^{\mathrm{BH}} \cos (n \phi)+K_{\mathrm{DVCS}} \sum_{n=0}^{2} c_{n}^{\mathrm{DVCS}} \cos (n \phi)\right\} .
\end{aligned}
$$

The asymmetries $\mathcal{A}_{\mathrm{LU}}^{\mathrm{I}}(\phi), \mathcal{A}_{\mathrm{LU}}^{\mathrm{DVCS}}(\phi)$, and $\mathcal{A}_{\mathrm{C}}(\phi)$ are related to the Fourier coefficients appearing in Eqs. 7-9, as illustrated by Eqs. 16-18. In analogy to the expansion of 
the cross section in Eq. 7-9, these asymmetries are also expanded in terms of the same harmonics in $\phi$ :

$$
\begin{aligned}
& \mathcal{A}_{\mathrm{LU}}^{\mathrm{I}}(\phi) \simeq \sum_{n=1}^{2} A_{\mathrm{LU}, \mathrm{I}}^{\sin (n \phi)} \sin (n \phi)+A_{\mathrm{LU}, \mathrm{I}}^{\cos (0 \phi)} \\
& \mathcal{A}_{\mathrm{LU}}^{\mathrm{DVCS}}(\phi) \simeq A_{\mathrm{LU}, \mathrm{DVCS}}^{\sin \phi} \sin \phi+A_{\mathrm{LU}, \mathrm{DVCS}}^{\cos (0 \phi)} \\
& \mathcal{A}_{\mathrm{C}}(\phi) \simeq \sum_{n=0}^{3} A_{\mathrm{C}}^{\cos (n \phi)} \cos (n \phi),
\end{aligned}
$$

where the approximation is due to the truncation of the in general infinite Fourier series caused by the azimuthal dependences in the denominators of Eqs. 16-18.

For each kinematic bin in $-t, x_{B}$, or $Q^{2}$, the sets of azimuthal asymmetry amplitudes $A_{\mathrm{LU}, \mathrm{I}}^{\sin (n \phi)}, A_{\mathrm{LU}, \mathrm{DVCS}}^{\sin \phi}$ and $A_{\mathrm{C}}^{\cos (n \phi)}$, hereafter called 'asymmetry amplitudes', are simultaneously extracted from the observed exclusive sample using the method of maximum likelihood (described in detail in Ref. [22]). Although these asymmetry amplitudes differ somewhat from the coefficients given in Eqs. 7-9 and Eqs. 16-18, they are well defined and can be computed in various GPD models for direct comparison with the data. Note that in Eqs. 33 and 34, an additional constant term $(n=0)$ was introduced as a consistency test. These terms must vanish as they are parity violating. Removing these constant terms or also introducing additional harmonic terms in the fitting procedure do not influence results for other asymmetry amplitudes [59].

\subsection{Background corrections and systematic uncertainties}

In each kinematic bin, the results from the maximum likelihood fit are corrected for photon background arising from semi-inclusive production of neutral mesons, mainly pions. A corrected asymmetry amplitude is obtained as

$$
A_{\text {corr }}=\frac{A_{\text {raw }}-f_{\text {semi }} \cdot A_{\text {semi }}}{1-f_{\text {semi }}} .
$$

Here, $A_{\text {raw }}$ stands for the extracted raw asymmetry amplitude, and $f_{\text {semi }}$ and $A_{\text {semi }}$ the fractional contribution and corresponding asymmetry amplitude of the semi-inclusive background. This fraction is obtained from a Monte Carlo simulation (see Section 4) and ranges from $1 \%$ to $11 \%$, depending on the kinematic conditions. As the semi-inclusive process is only very weakly beam-charge dependent, its asymmetry with respect to the beam charge or to the product of the beam charge and the beam polarization is assumed to be zero. The asymmetry of the semi-inclusive $\pi^{0}$ background with respect to only the longitudinal beam polarization is extracted from experimental data by requiring two photons to be detected in the calorimeter with an invariant mass between $0.10 \mathrm{GeV}$ and $0.17 \mathrm{GeV}$ and with no associated charged tracks. The restriction on the energy deposition in the calorimeter of the less energetic cluster is relaxed to $1 \mathrm{GeV}$ to improve the statistical precision. The fractional energy $z=E_{\pi} / \nu$ of the reconstructed neutral pions is required to be larger than 0.8. After applying the correction of Eq. 36, the resulting asymmetry amplitudes are expected to originate from elastic (coherent), and incoherent photon production possibly including nucleon excitation. 
Table 2

The main contributions to the systematic uncertainty of extracted asymmetry amplitudes of interest, averaged over the full kinematic range. Not included is a $2.4 \%$ scale uncertainty of the beam-helicity asymmetries due to the beam polarization measurement.

\begin{tabular}{crcc}
\hline Amplitude & $M_{X}^{2}$ shift & Background corr. & Acceptance, smearing, bin width, alignment \\
\hline$A_{\mathrm{C}}^{\cos (0 \phi)}$ & 0.001 & 0.001 & 0.014 \\
$A_{\mathrm{C}}^{\cos \phi}$ & $<0.001$ & 0.002 & 0.023 \\
$A_{\mathrm{LU}, \mathrm{I}}^{\sin \phi}$ & $<0.001$ & 0.004 & 0.031 \\
$A_{\mathrm{LU}, \mathrm{DVCS}}^{\sin \phi}$ & 0.002 & 0.006 & 0.003 \\
\hline
\end{tabular}

The combined contribution to the systematic uncertainty from detector acceptance, smearing, finite bin width, and alignment of the detector elements with respect to the beam is determined from a Monte Carlo simulation using the GPD model described in Ref. [60]. Note that a mistake has been found in this GPD model [61]; however, the model described previously reported HERMES beam-charge [22] and preliminary (singlecharge) beam-helicity asymmetries well [62] and thus is considered to be adequate for systematic studies. In each bin, the systematic uncertainty is taken as the difference between the model prediction at the mean kinematic value of that bin and the respective amplitude extracted from the reconstructed Monte Carlo data. The dominant contributions to the total systematic uncertainty are those from the detector acceptance and finite bin width. Further sources of uncertainty are associated with the background correction and a relative shift of the $M_{X}^{2}$ spectra between the data samples from various running periods [22]. The contributions to the systematic uncertainty are added in quadrature. The main contributions for asymmetry amplitudes of interest are given in Table 2. Not included is any contribution due to additional QED vertices, as the most significant of these has been estimated to be negligible [63].

\section{Results}

\subsection{Results on beam-charge and beam-helicity asymmetries for an unpolarized deuterium target}

The asymmetry amplitudes are shown in Figs. $6-8$ as a function of $-t, x_{N}$, or $Q^{2}$. While the variable $x_{D}$ would be the appropriate choice when presenting experimental results for pure coherent scattering, the nucleonic Bjorken variable $x_{N}$ is the practical choice in this case where incoherent scattering dominates over most of the kinematic range. The variables $x_{N}$ and $Q^{2}$ are strongly correlated due to the experimental acceptance. The 'overall' results in the left columns correspond to the entire HERMES kinematic acceptance. Figure 6 shows the amplitudes $A_{\mathrm{C}}^{\cos (n \phi)}$, which are related to beam charge only, and Fig. 7 shows the amplitude $A_{\mathrm{LU}, \mathrm{DVCS}}^{\sin \phi}$, which is related to beam helicity only, and the amplitudes $A_{\mathrm{LU}, \mathrm{I}}^{\sin (n \phi)}$, which are related to both. All amplitudes are listed in Table 3 with the mean kinematic values of each bin ${ }^{17}$.

\footnotetext{
$\overline{17}$ These results for only four bins in $-t, x_{N}$, or $Q^{2}$, i.e., a binning used in previous HERMES papers $[22,23]$, are available in the Durham database.
} 


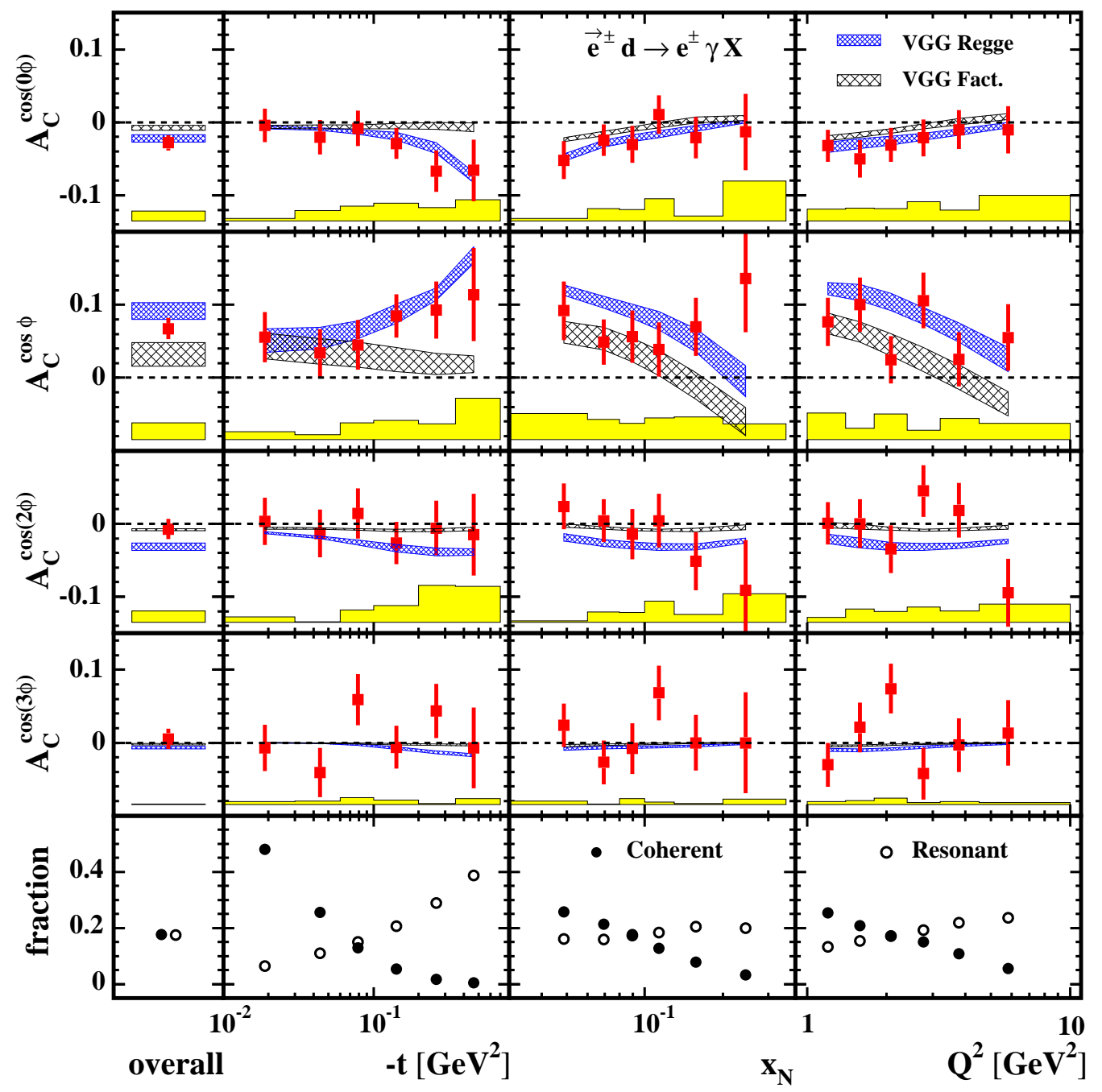

Fig. 6. Amplitudes of the beam-charge asymmetry, which are sensitive to the interference term, in bins of $-t, x_{N}$, or $Q^{2}$. The squares represent the results from the present work. The error bars (bands) represent the statistical (systematic) uncertainties. The finely (coarsely) hatched bands are theoretical calculations for incoherently combined proton and neutron targets, using variants of a double-distribution model $[54,66,64]$ with the VGG Regge (VGG Factorized) ansatz for GPDs. The lowest panel shows the simulated fractions of coherent and resonant production.

Of special interest is the asymmetry amplitude $A_{\mathrm{C}}^{\cos \phi}$, which is sensitive to the GPD $H_{1}$ $(H)$ for the coherent (incoherent) process in HERMES kinematic conditions (see Eqs. 29 and 24). The present data indicates that this amplitude increases with increasing $-t$. The amplitude $A_{\mathrm{C}}^{\cos (0 \phi)}$ in Fig. 6, which is expected to relate to the same combination of GPDs as does $A_{\mathrm{C}}^{\cos \phi}$, shows similar behaviour but with opposite sign, as expected [15]. The other two amplitudes $A_{\mathrm{C}}^{\cos (2 \phi)}$ and $A_{\mathrm{C}}^{\cos (3 \phi)}$, related to twist-3 GPDs and the gluon transversity operator, respectively (see Section 2.2 ), are consistent with zero. 


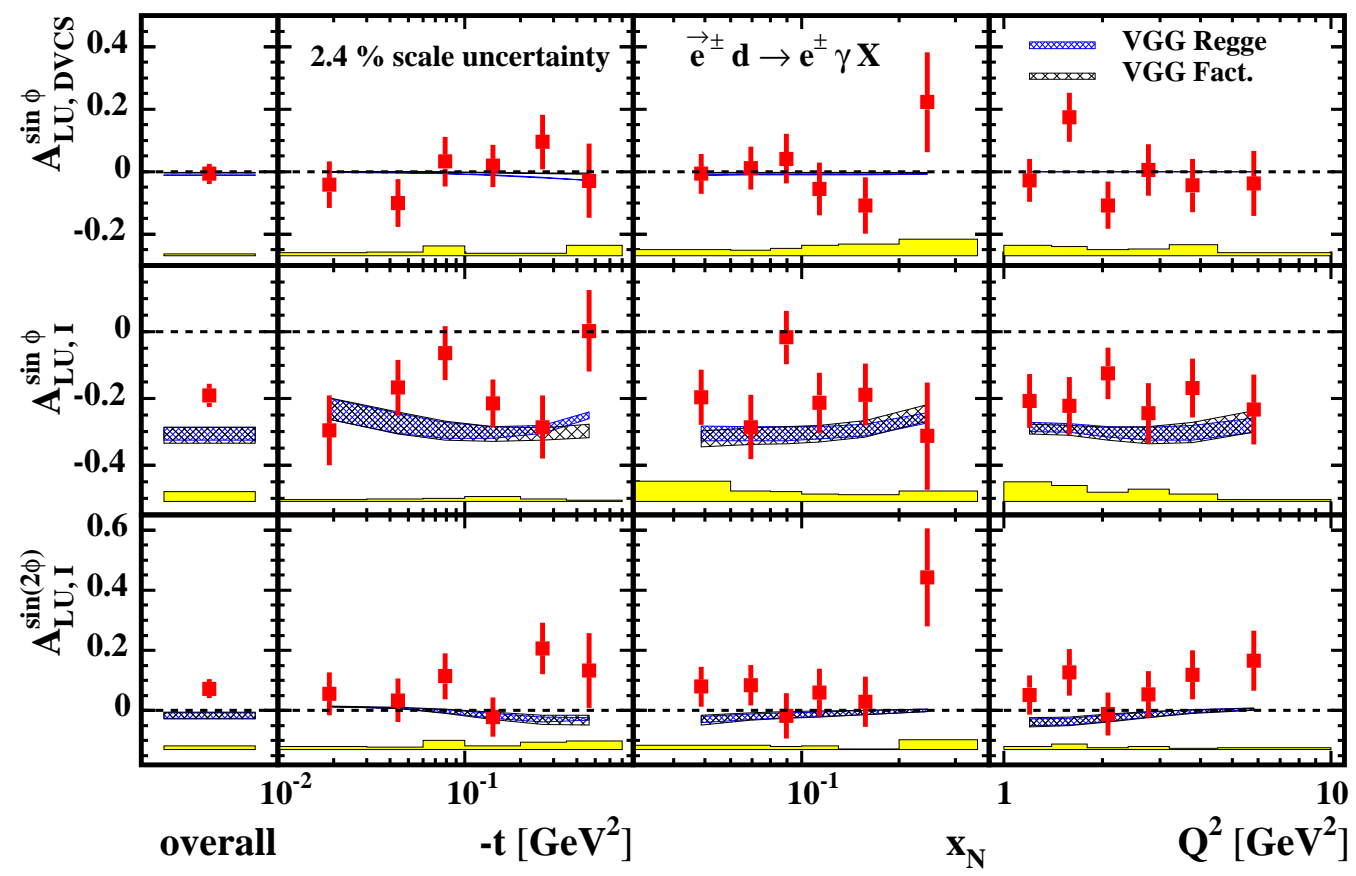

Fig. 7. The first row shows the $\sin \phi$ amplitude of the beam-helicity asymmetry that is sensitive to the squared DVCS term, in bins of $-t, x_{N}$, or $Q^{2}$. Correspondingly, the second (third) row shows the $\sin \phi(\sin 2 \phi)$ amplitude of the beam-helicity asymmetry sensitive to the interference term. All symbols are defined as in Fig. 6. There is an overall 2.4\% scale uncertainty arising from the uncertainty in the measurement of the beam polarization.

The fractional contributions to the yield from the coherent processes and from processes with excitation of resonant final states are presented in the bottom row of Fig. 6 (see also Table 4), as obtained from the Monte Carlo simulation using the exclusive-photon generator mentioned in Section 4. Note that these fractional contributions are subject to considerable model dependence.

Figure 7 shows amplitudes of beam-helicity asymmetries, with the charge-averaged case related to the squared DVCS term in the upper row and the charge-difference case related to the interference term in the other rows. The amplitude $A_{\mathrm{LU}, \mathrm{DVCS}}^{\sin \phi}$, which is related to twist-3 GPDs, is found to be consistent with zero. Like the amplitude $A_{\mathrm{C}}^{\cos \phi}$, the amplitude $A_{\mathrm{LU}, \mathrm{I}}^{\mathrm{sin} \phi}$ is also sensitive to the GPD $H_{1}[H]$ for the coherent [incoherent] process, although these two asymmetries reveal different aspects of the (real) GPD, selected by different convolutions with (complex) hard scattering amplitudes. While the amplitude $A_{\mathrm{C}}^{\cos \phi}$ is related to the real part of the $\mathrm{CFF} \mathcal{H}_{1}[\mathcal{H}]$, the $A_{\mathrm{LU}, \mathrm{I}}^{\sin \phi}$ amplitude is proportional to the imaginary part and shows significant negative values. The amplitude $A_{\mathrm{LU}, \mathrm{I}}^{\sin (2 \phi)}$ appears at twist-3 level, but nevertheless it shows a value which is non-zero and positive by 1.7 standard deviations of the total experimental uncertainty. Figure 8 shows the amplitudes that are forbidden by parity conservation but were included in the fit as a consistency test. They are consistent with zero.

The two hatched bands in Figs. 6 and 7 are theoretical calculations for the incoherent 
Table 3

Results for azimuthal Fourier amplitudes of the asymmetries with respect to the beam charge and helicity for the exclusive sample.

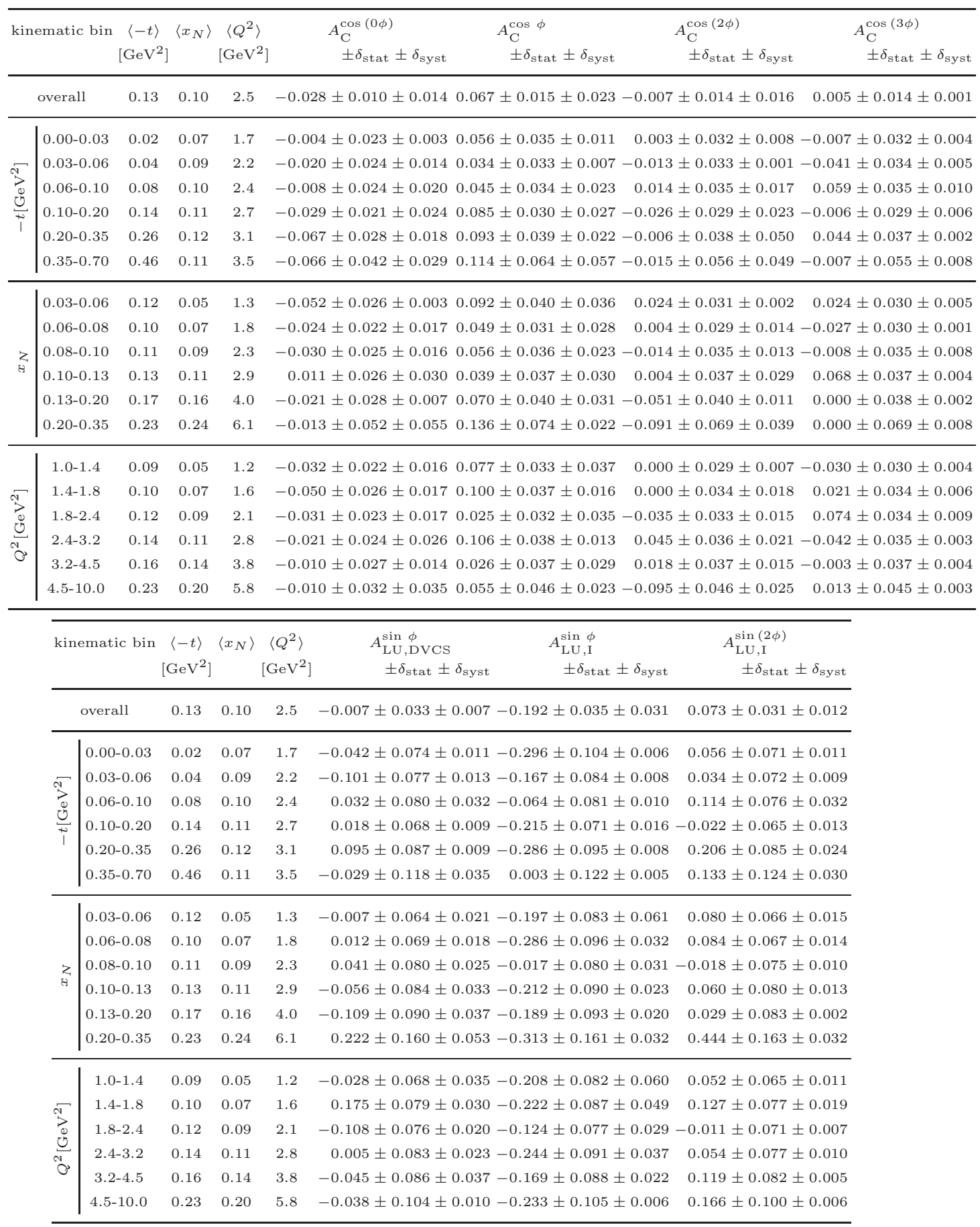




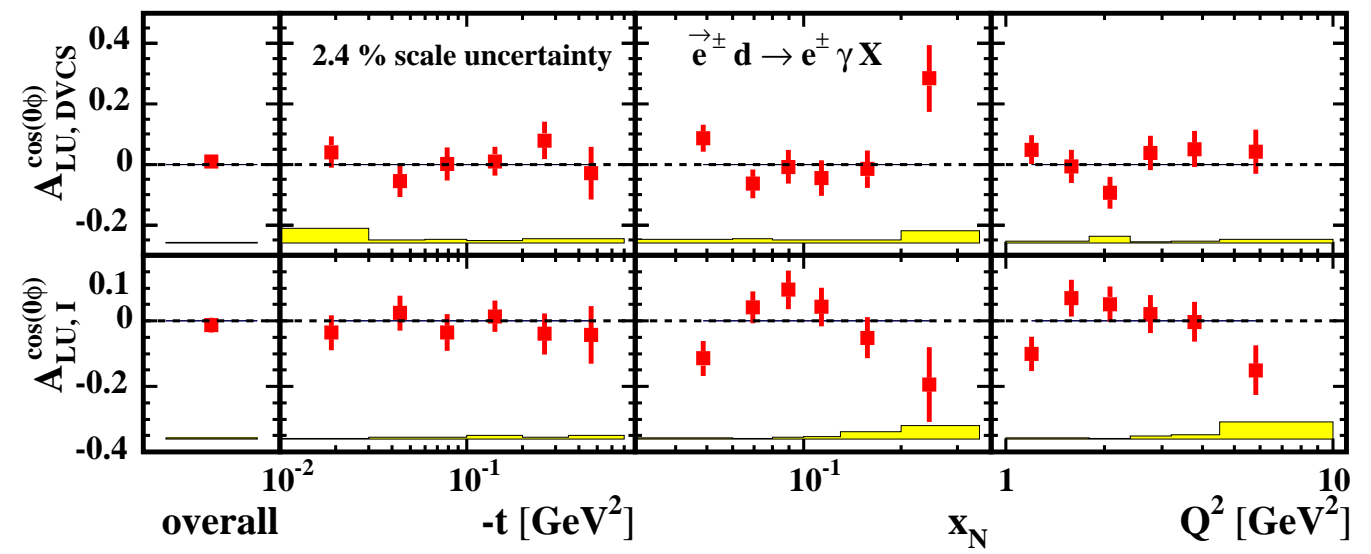

Fig. 8. The $\cos (0 \phi)$ amplitudes (constant terms) that are included as a consistency test in the fit in Eqs. 34 and 33. All symbols are defined as in Fig. 6. There is an overall $2.4 \%$ scale uncertainty arising from the uncertainty in the measurement of the beam polarization.

process, based on two different ansätze for modeling GPDs [64] in the VGG model [65] (the coherent process will be considered in Section 6.3.). In this model, a GPD is written as a double distribution $[2,3]$ complemented by a D-term $[37,38]$ :

- In the 'factorized ansatz' (VGG Fact.), the dependences on $t$ and $(x, \xi)$ are uncorrelated. The $t$ dependence is written in accordance with proton elastic form factors. The $(x, \xi)$ dependence is based on double distributions [2] constructed from ordinary PDFs complemented with a profile function that characterizes the strength of the $\xi$ dependence; in the limit $b \rightarrow \infty$ of the profile parameter $b$, the GPD is independent of $\xi[66]$. Note that $b$ is a free parameter to be experimentally determined independently for valence and sea quarks.

- The 'Regge ansatz' (VGG Regge) implements entanglement of the $t$ dependence of the GPD with its dependence on $x$ and $\xi$. This feature is inspired by the traditional interpretation of measurements of elastic diffractive processes in terms of Regge phenomenology [64], and finds further support in more recent phenomenological considerations $[67,68]$. This ansatz for GPDs hence uses for the $t$ dependence of the double distributions a soft Regge-type parameterization $\propto|\xi|^{-\alpha(0)+\alpha^{\prime}|t|}$ with $\alpha^{\prime}=$ $0.8 \mathrm{GeV}^{-2} \ldots 0.9 \mathrm{GeV}^{-2}$ for quarks.

Both theoretical calculations are averaged at the cross section level over incoherent processes on the proton and neutron in each kinematic bin. In both calculations the D-term is assigned the value zero. Earlier, it was found that inclusion of a D-term with any significant magnitude in the double-distribution model of Ref. [65] employing several variants of Regge or factorized ansätze with any choice of profile parameters fails to describe the BCA amplitudes measured at HERMES on a hydrogen target $[22,23]$. The theoretical bands in Figs. 6 and 7 correspond to the range of values of the asymmetry amplitudes obtained by varying the profile parameters $b_{\text {val }}$ and $b_{\text {sea }}$ between unity and infinity. The theoretical calculations based on the factorized ansatz fail to describe the $t$ dependence of $A_{\mathrm{C}}^{\cos (0 \phi)}$ and $A_{\mathrm{C}}^{\cos \phi}$ as seen in Fig. 6. The calculations based on the Regge ansatz for GPDs are in good agreement with the $t$ dependence of the measured asymmetry amplitudes with respect to the beam charge $A_{\mathrm{C}}^{\cos (n \phi)}$. Both ansätze predict 
Table 4

Simulated fractional contributions for coherent and resonant processes in each kinematic bin.

\begin{tabular}{|c|c|c|c|}
\hline \multicolumn{2}{|c|}{ kinematic bin } & coherent & resonant \\
\hline & overall & 0.176 & 0.174 \\
\hline \multirow{6}{*}{ 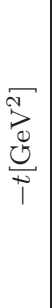 } & $0.00-0.03$ & 0.481 & 0.064 \\
\hline & $0.03-0.06$ & 0.256 & 0.110 \\
\hline & $0.06-0.10$ & 0.130 & 0.150 \\
\hline & $0.10-0.20$ & 0.053 & 0.206 \\
\hline & $0.20-0.35$ & 0.017 & 0.289 \\
\hline & $0.35-0.70$ & 0.005 & 0.387 \\
\hline \multirow{6}{*}{$z_{8}^{Z}$} & $0.03-0.06$ & 0.258 & 0.161 \\
\hline & $0.06-0.08$ & 0.214 & 0.160 \\
\hline & $0.08-0.10$ & 0.176 & 0.173 \\
\hline & $0.10-0.13$ & 0.127 & 0.184 \\
\hline & $0.13-0.20$ & 0.078 & 0.203 \\
\hline & $0.20-0.35$ & 0.032 & 0.198 \\
\hline \multirow{6}{*}{ 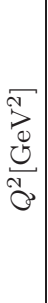 } & $1.0-1.4$ & 0.253 & 0.133 \\
\hline & $1.4-1.8$ & 0.209 & 0.154 \\
\hline & $1.8-2.4$ & 0.172 & 0.172 \\
\hline & $2.4-3.2$ & 0.150 & 0.193 \\
\hline & $3.2-4.5$ & 0.109 & 0.219 \\
\hline & $4.5-10.0$ & 0.055 & 0.237 \\
\hline
\end{tabular}

that $A_{\mathrm{C}}^{\cos \phi}$ decreases with increasing $x_{N}$, which is not seen in the data. Both ansätze undershoot the asymmetry amplitudes with respect to the beam helicity $A_{\mathrm{LU}, \mathrm{I}}^{\sin (n \phi)}$.

6.2. Comparison of the deuteron results with the HERMES results on beam-charge and beam-helicity asymmetries on the proton

In Figs. 9-11 the overall asymmetry amplitudes as well as their $-t, x_{N}$, and $Q^{2}$ dependences, measured for the unpolarized deuterium target, are compared with the analogous results obtained from HERMES data on the proton [27].

The deuteron data include the coherent process $e^{ \pm} d \rightarrow e^{ \pm} d \gamma$, and the incoherent process $e^{ \pm} d \rightarrow e^{ \pm} p n \gamma$, where a nucleon may be excited to a resonance. The proton data include only $\boldsymbol{e}^{ \pm} p \rightarrow e^{ \pm} p \gamma$ and the case with resonance excitation. Any difference that appears at small values of $-t$ may be due to the coherent process. Monte Carlo simulations indicate that the incoherent process dominates for $0.06 \mathrm{GeV}^{2}<-t<0.7 \mathrm{GeV}^{2}$ (see Fig. 5). As shown in Figs. 9-11, the deuteron and proton results are found to be consistent in most kinematic regions. A possible difference in the last two $-t$ bins of the amplitude 


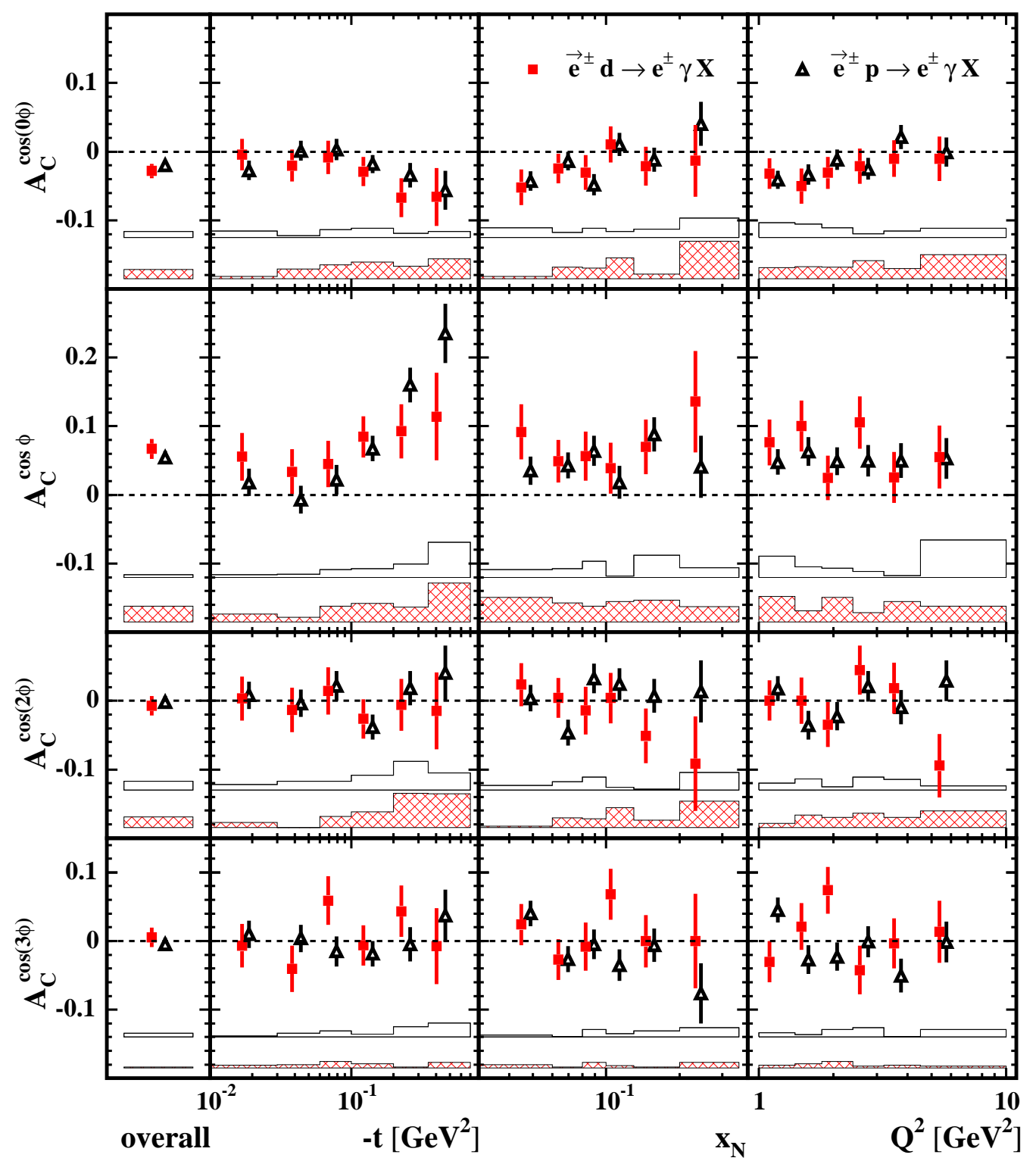

Fig. 9. Fourier amplitudes of the beam-charge asymmetry, which are sensitive to the interference term, in bins of $-t, x_{N}$, or $Q^{2}$, extracted from deuteron data (squares) and from proton data (triangles). The points for deuterium are slightly shifted along the x-axis for visibility. The error bars (bands) represent the statistical (systematic) uncertainties. The hatched band is for the deuterium target.

$A_{\mathrm{C}}^{\cos \phi}$ (see Fig. 9) may be due to the contributions of the neutron and its resonances. The proton and deuteron results for the amplitude $A_{\mathrm{LU}, \mathrm{I}}^{\sin (2 \phi)}$ integrated over the acceptance differ by 2.5 times the total experimental uncertainties. This possible discrepancy is most evident at large $-t$ and large $x_{N}$ (or $Q^{2}$ ). Such a discrepancy would have no obvious explanation. 


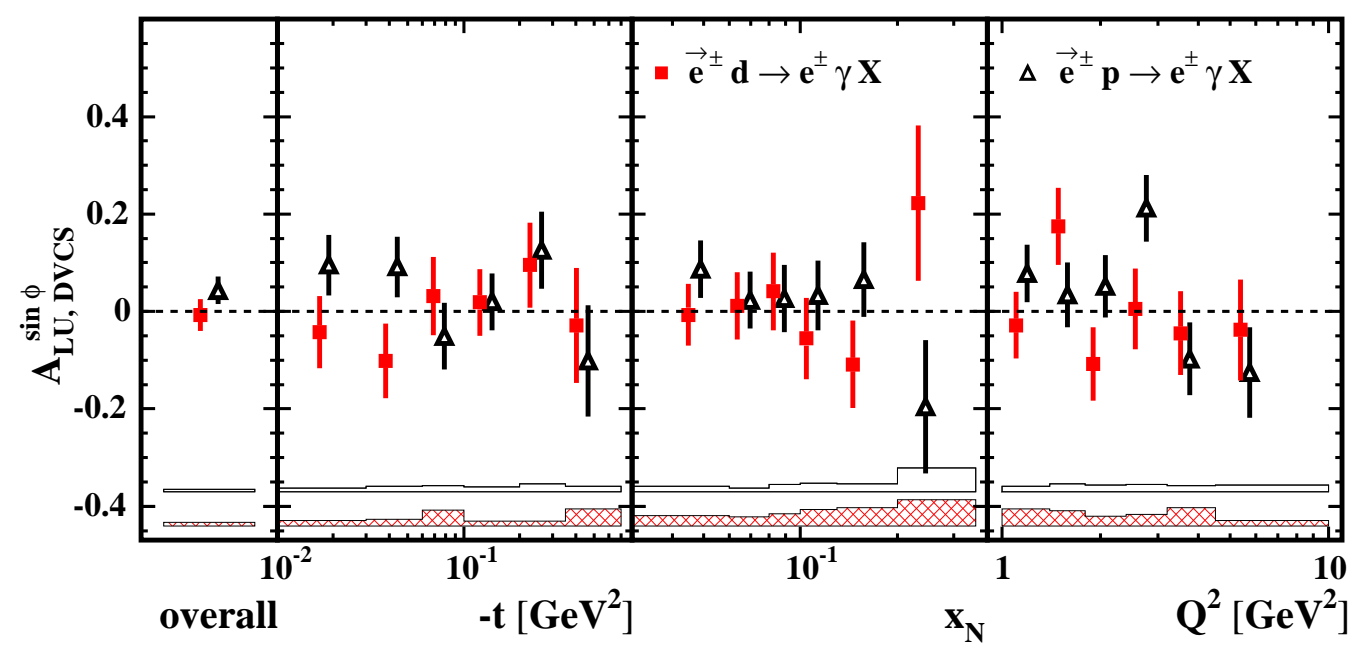

Fig. 10. Fourier amplitudes of the beam-helicity asymmetry that are sensitive to the squared DVCS term, in bins of $-t, x_{N}$, or $Q^{2}$, extracted from deuteron data (squares) and from proton data (triangles). The error bars (bands) represent the statistical (systematic) uncertainties, which include all sources apart from the $2.4 \%(2.8 \%)$ scale uncertainty for the deuteron (proton) data due to the beam polarization. The hatched band is for the deuterium target.

\subsection{Estimates of the asymmetries from coherent scattering}

Estimates of the asymmetries for coherent scattering in the range $-t<0.06 \mathrm{GeV}^{2}$, corresponding to the first two bins, were derived by correcting for the incoherent contributions of the proton and its resonances using the simulated fractional coherent contributions from Table 4, under the assumption that the asymmetries for these contributions are the same as those on the free proton. The simulated contribution of approximately $7 \%$ from the process $e^{ \pm} n \rightarrow e^{ \pm} n \gamma$ is estimated to have an effect on the asymmetries of less than 0.01. The extracted coherent asymmetries $A_{\mathrm{C}, \mathrm{coh}}^{\cos \phi}$ and $A_{\mathrm{LU}, \mathrm{I}, \mathrm{coh}}^{\sin \phi}$ are found to be $0.11 \pm 0.07$ (stat.) \pm 0.03 (syst.) and $-0.29 \pm 0.18$ (stat.) \pm 0.03 (syst.), respectively, at the average kinematic values ${ }^{18}\langle-t\rangle=0.03 \mathrm{GeV}^{2},\left\langle x_{D}\right\rangle=0.04$, and $\left\langle Q^{2}\right\rangle=1.9 \mathrm{GeV}^{2}$.

These results for the coherent asymmetries are compared in Table 5 with model estimates using the models $A, B, B_{0}, \widehat{B}, B^{\prime}$, and $C$ of Refs. [15,35], the main parameters of which are listed in Table 6 . The model estimates are based on the double distribution ansatz [66] for nucleonic GPDs, combined with a factorized $t$ dependence, and with the D-term set to zero. The nucleonic GPDs are combined using the impulse approximation. The contribution of sea quarks is neglected in model $B_{0}$, while it is enhanced in model $C$ by a choice of a smaller value of the parameter $b_{\text {sea }}$, which increases the absolute value of the beam-helicity asymmetry amplitude $A_{\mathrm{LU}, \mathrm{I}, \mathrm{coh}}^{\mathrm{sin} \phi}$ compared to model $A$. In model $B^{\prime}(\widehat{B})$, the GPD $H_{3}\left(H_{5}\right)$ is taken into account by arbitrarily equating it with $H_{1}\left(H_{1}(x)-H_{1}(-x)\right)$. All other GPDs are kinematically suppressed and are set to zero. The models $B_{0}$ and $C$ were previously ruled out by the beam-helicity and beam-charge asymmetry measurements on the hydrogen target $[18,19,22,23,27]$.

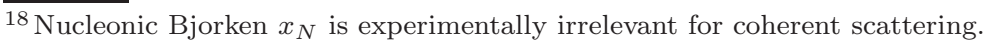




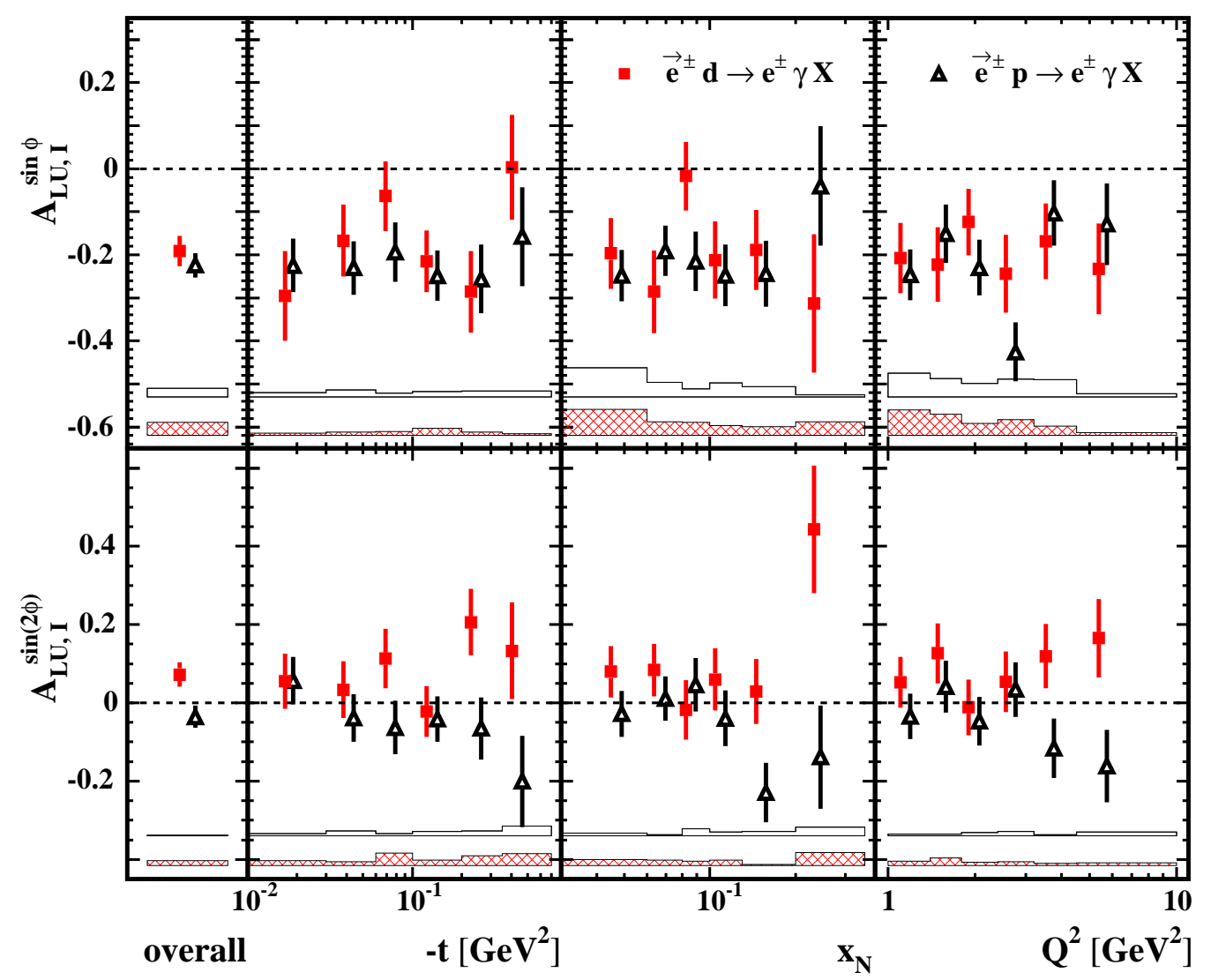

Fig. 11. Fourier amplitudes of the beam-helicity asymmetry that are sensitive to the interference term, in bins of $-t, x_{N}$, or $Q^{2}$, extracted from deuteron data (squares) and from proton data (triangles). The error bars (bands) represent the statistical (systematic) uncertainties, which include all sources apart from the $2.4 \%(2.8 \%)$ scale uncertainty for the deuteron (proton) data due to the beam polarization. The hatched band is for the deuterium target.

Table 5 also includes model predictions from Ref. [38]. This model is based on double distributions, where only the polarizations of the valence quarks are considered for the nucleonic GPDs. A factorized ansatz for the $t$ dependence of the nucleonic GPDs is employed and the strange quark contribution is neglected. Again the impulse approximation is used to combine the nucleonic GPDs, without including the particular contribution from the D-term.

All models are consistent within two standard deviations in the total experimental uncertainty with the extracted results for $A_{\mathrm{LU} \text {,I,coh }}^{\sin \phi}$ and $A_{\mathrm{C}, \mathrm{coh}}^{\cos \phi}$, except for models $B_{0}$ and that of Ref. [38], which disagree with the results of $A_{\mathrm{C}, \mathrm{coh}}^{\cos \phi}$ by about 3.5 standard deviations. Here, it should be noted that predictions for the real part of the CFFs are subject to delicate cancellations [15] and hence are extremely sensitive to assumptions. 
Table 5

Experimental and theoretical values of the beam-helicity and beam-charge asymmetries for the coherent process on the deuteron. The theoretical predictions are for variants of the models of Ref. [15,35] and a model from Ref. [38]. The experimental uncertainties do not account for the model dependence of the simulated fractional contributions of coherent and incoherent processes.

\begin{tabular}{lrrrrrrrr}
\hline & Exp. value & \multicolumn{7}{c}{ Model } \\
\hline & value $\pm \delta_{\text {stat }} \pm \delta_{\text {syst }}$ & $\mathrm{A}$ & $\mathrm{B}$ & $\mathrm{B}_{0}$ & $\widehat{B}$ & $B^{\prime}$ & $\mathrm{C}$ & {$[38]$} \\
\hline$A_{\mathrm{LU}, \mathrm{I}, \mathrm{coh}}^{\text {sin }}$ & $-0.29 \pm 0.18 \pm 0.03$ & -0.44 & -0.38 & -0.16 & -0.37 & -0.39 & -0.58 & -0.36 \\
$A_{\mathrm{C}, \mathrm{coh}}^{\text {cos } \phi}$ & $0.11 \pm 0.07 \pm 0.03$ & 0.10 & 0.09 & -0.17 & 0.09 & 0.09 & 0.22 & -0.15 \\
\hline
\end{tabular}

Table 6

Model parameter sets for the GPD $H_{1}$ of the deuteron $[15,35]$. The $t$ slope parameter $B_{\text {sea }}$ is used mainly to change the normalization of the sea quark GPD $H_{1}$.

\begin{tabular}{ccccc}
\hline deuteron $H_{1}$ GPD & \multicolumn{5}{c}{ Model } \\
\hline Model parameters & $\mathrm{A}$ & $\mathrm{B}\left(B^{\prime}, \widehat{B}\right)$ & $\mathrm{B}_{0}$ & $\mathrm{C}$ \\
\hline$b_{\text {val }}$ & 1 & $\infty$ & $\infty$ & 1 \\
$b_{\text {sea }}$ & $\infty$ & $\infty$ & - & 1 \\
$B_{\text {sea }}\left[\mathrm{GeV}^{-2}\right]$ & 20 & 20 & - & 15 \\
\hline
\end{tabular}

\section{Summary}

Azimuthal asymmetries with respect to beam-helicity and beam-charge are measured for hard exclusive electroproduction of photons in deeply inelastic scattering off an unpolarized deuterium target. The observed asymmetries are attributed to either the interference between the DVCS and the Bethe-Heitler processes or the pure DVCS process. The asymmetries are observed in the exclusive missing-mass domain $-(1.5)^{2} \mathrm{GeV}^{2}<M_{X}^{2}<$ $(1.7)^{2} \mathrm{GeV}^{2}$. The dependences of these asymmetries on $-t, x_{N}$, or $Q^{2}$ are investigated. The results from the deuterium target include the coherent process $\boldsymbol{e}^{ \pm} d \rightarrow e^{ \pm} d \gamma$ and the incoherent process $\boldsymbol{e}^{ \pm} d \rightarrow e^{ \pm} p n \gamma$, where a nucleon may be excited to a resonance. For an unpolarized deuterium target, the leading Fourier amplitude of the beam-helicity asymmetry that is sensitive to the interference term is found to be substantial, but no significant $t$ dependence is observed. The leading amplitude of the beam-charge asymmetry is substantial at large $-t$, but becomes small at small values of $-t$. The amplitudes of the beam-helicity asymmetry that are sensitive to the squared DVCS term are found to be consistent with zero. The data are able to discriminate among various GPD models.

The measured asymmetry amplitudes from unpolarized deuteron and proton [27] targets are consistent in most kinematic regions, except possibly for the leading amplitude of the beam-charge asymmetry in the last two $-t$ bins, and the 'overall' value of $A_{\mathrm{LU}}^{\sin (2 \phi)}$.

The beam-charge and beam-helicity asymmetry amplitudes for coherent scattering from the deuteron are extracted from the asymmetry amplitudes measured on unpolarized deuteron and proton targets. When compared to the GPD models of Refs. [15,35], the results disfavor a large sea quark contribution while favoring a non-zero contribution. The results disfavor the variants of the model of Refs. $[15,35]$ that omit sea quark contributions, and also the model of Ref. [38]. 


\section{Acknowledgments}

We gratefully acknowledge the DESY management for its support and the staff at DESY and the collaborating institutions for their significant effort. This work was supported by the FWO-Flanders and IWT, Belgium; the Natural Sciences and Engineering Research Council of Canada; the National Natural Science Foundation of China; the Alexander von Humboldt Stiftung; the German Bundesministerium für Bildung und Forschung (BMBF); the Deutsche Forschungsgemeinschaft (DFG); the Italian Istituto Nazionale di Fisica Nucleare (INFN); the MEXT, JSPS, and G-COE of Japan; the Dutch Foundation for Fundamenteel Onderzoek der Materie (FOM); the U.K. Engineering and Physical Sciences Research Council, the Science and Technology Facilities Council, and the Scottish Universities Physics Alliance; the U.S. Department of Energy (DOE) and the National Science Foundation (NSF); the Russian Academy of Science and the Russian Federal Agency for Science and Innovations; the Ministry of Economy and the Ministry of Education and Science of Armenia; and the European Community-Research Infrastructure Activity under the FP6 "Structuring the European Research Area" program (HadronPhysics, contract number RII3-CT-2004-506078).

\section{References}

[1] M. Burkardt, A. Miller, and W.D. Nowak, DESY-08-162, JLAB-THY-08-913, arXiv:0812.2208 [hep$\mathrm{ph}]$.

[2] D. Müller et al., Fortsch. Phys. 42 (1994) 101.

[3] A.V. Radyushkin, Phys. Lett. B380 (1996) 417. A.V. Radyushkin, Phys. Rev. D56 (1997) 5524.

[4] X. Ji, Phys. Rev. Lett. 78 (1997) 610.

X. Ji, Phys. Rev. D55 (1997) 7114.

[5] J.P. Ralston and B. Pire, Phys. Rev. D66 (2002) 111501.

[6] M. Burkardt, Phys. Rev. D62 (2000) 071503; Erratum-ibid D66 (2002) 119903. M. Burkardt, Int. J. Mod. Phys. A18 (2003) 173.

[7] M. Diehl, Eur. Phys. J. C25 (2002) 223; Erratum-ibid C31 (2003) 277.

[8] A.V. Belitsky and D. Müller, Nucl. Phys. A711 (2002) 118.

[9] M. Diehl, Phys. Rept. 388 (2003) 41.

[10] A.V. Belitsky and A.V. Radyushkin, Phys. Rept. 418 (2005) 1.

[11] A.V. Belitsky and D. Müller, Phys. Lett. B417 (1998) 129.

[12] X. Ji and J. Osborne, Phys. Rev. D58 (1998) 094018.

[13] L. Mankiewicz et al., Phys. Lett. B425 (1998) 186.

[14] N. Kivel, M.V. Polyakov, and M. Vanderhaeghen, Phys. Rev. D63 (2001) 114014.

[15] A.V. Belitsky, D. Müller, and A. Kirchner, Nucl. Phys. B629 (2002) 323.

[16] A. Freund, Phys. Rev. D68 (2003) 096006.

[17] A.V. Belitsky, A. Freund, and D. Müller, Nucl. Phys. B574 (2000) 347.

[18] HERMES Collaboration, A. Airapetian et al., Phys. Rev. Lett. 87 (2001) 182001.

[19] CLAS Collaboration, S. Stepanyan et al., Phys. Rev. Lett. 87 (2001) 182002.

[20] M. Kopytin, for the HERMES Collaboration, AIP Conf. Proc. 792 (2005) 424.

[21] CLAS Collaboration, S. Chen et al., Phys. Rev. Lett. 97 (2006) 072002.

[22] HERMES Collaboration, A. Airapetian et al., JHEP 06 (2008) 066.

[23] HERMES Collaboration, A. Airapetian et al., Phys. Rev. D75 (2007) 011103.

[24] JLAB Hall A Collaboration, C.M. Camacho et al., Phys. Rev. Lett. 97 (2006) 262002.

[25] CLAS Collaboration, F.X. Girod et al., Phys. Rev. Lett. 100 (2008) 162002.

[26] CLAS Collaboration, G. Gavalian et al., Phys. Rev. C80 (2009) 035206.

[27] HERMES Collaboration, A. Airapetian et al., JHEP 11 (2009) 083. 
[28] HERMES Collaboration, A. Airapetian et al., DESY-09-190, arXiv:0911.0091 [hep-ex].

[29] M. V. Polyakov, Phys. Lett. B555 (2003) 57.

[30] J.C. Collins and A. Freund, Phys. Rev. D59 (1999) 074009.

[31] J. Blümlein, B. Geyer, and D. Robaschik, Phys. Lett. B406 (1997) 161. J. Blümlein, B. Geyer, and D. Robaschik, Nucl. Phys. B560 (1999) 283.

[32] E.R. Berger, F. Cano, M. Diehl, and B. Pire, Phys. Rev. Lett. 87 (2001) 142302.

[33] HERMES Collaboration, A. Airapetian et al., Phys. Rev. Lett. 95 (2005) 242001.

[34] M. Lacombe et al., Phys. Lett. B101 (1981) 139.

[35] A. Kirchner and D. Müller, Eur. Phys. J. C32 (2003) 347.

[36] M. Diehl, T. Gousset, B. Pire, and J.P. Ralston, Phys. Lett. B411 (1997) 193.

[37] M.V. Polyakov and C. Weiss, Phys. Rev. D60 (1999) 114017.

[38] F. Cano and B. Pire, Eur. Phys. J. A19 (2004) 423.

[39] P. Hoodbhoy and X. Ji, Phys. Rev. D58 (1998) 054006.

[40] A. Belitsky and D. Müller, Phys. Lett. B486 (2000) 369.

[41] M. Diehl et al., Eur. Phys. J. C19 (2001) 485.

[42] N. Kivel and L. Mankiewicz, Eur. Phys. J. C21 (2001) 621.

[43] JLAB t20 Collaboration, D. Abbott et al., Eur. Phys. J. A7 (2000) 421.

[44] HERMES Collaboration, K. Ackerstaff et al., Nucl. Instr. and Meth. A417 (1998) 230.

[45] A. Sokolov and I. Ternov, Sov. Phys. Doklady 8 (1964) 1203.

[46] J. Buon and K. Steffen, Nucl. Instr. and Meth. A245 (1986) 248.

[47] D.P. Barber et al., Nucl. Instr. and Meth. A338 (1994) 166.

[48] M. Beckmann et al., Nucl. Instr. and Meth. A479 (2002) 334.

[49] B. Krauss, PhD thesis, Friedrich-Alexander Universität Erlangen-Nürnberg, Germany, February 2005, DESY-THESIS-2005-008.

[50] V.A. Korotkov and W.D. Nowak, Eur. Phys. J. C23 (2002) 455.

[51] G. Ingelmann, A. Edin, and J. Rathsman, Comput. Phys. Commun. 101 (1997) 108.

[52] T. Sjöstrand, Comput. Phys. Commun. 82 (1994) 74.

[53] A. Hillenbrand, PhD thesis, Friedrich-Alexander Universität Erlangen-Nürnberg, Germany, November 2005, DESY-THESIS-2005-035.

[54] M. Vanderhaeghen, P.A.M. Guichon, and M. Guidal, Phys. Rev. D60 (1999) 094017.

[55] Z. Ye, PhD thesis, Universität Hamburg, Germany, December 2006, DESY-THESIS-2007-005.

[56] A. Vandenbroucke, PhD thesis, Universiteit Gent, Belgium, November 2006, DESY-THESIS-2007003.

[57] M. Vanderhaeghen et al., Phys. Rev. C62 (2000) 025501.

[58] F. Ellinghaus, PhD thesis, Humboldt Universität Berlin, Germany, January 2004, DESY-THESIS2004-005.

[59] G.D. Hill, PhD thesis, University of Glasgow, Scotland, August 2008, DESY-THESIS-2008-023.

[60] V. Guzey and T. Teckentrup, Phys. Rev. D74 (2006) 054027.

[61] V. Guzey and T. Teckentrup, Phys. Rev. D79 (2009) 017501.

[62] F. Ellinghaus (for the HERMES collaboration), Proceedings of the Workshop on "Exclusive Reactions at High Momentum Transfer", Jefferson Lab, Newport News, USA, May 2007, arXiv:0710.5768.

[63] A.V. Afanasev, M.I. Konchatnij, and N.P. Merenkov, J. Exp. Theor. Phys. 102 (2006) 220.

[64] K. Goeke, M.V. Polyakov, and M. Vanderhaeghen, Prog. Part. Nucl. Phys. 47 (2001) 401.

[65] M. Vanderhaeghen, P.A.M. Guichon, and M. Guidal, Computer code for the calculation of DVCS and $\mathrm{BH}$ processes in the reaction $e p \rightarrow e p \gamma$, private communication, 2001.

[66] A.V. Radyushkin, Phys. Rev. D59 (1999) 014030.

[67] M. Diehl, Th. Feldmann, R. Jakob, and P. Kroll, Eur. Phys. J. C39 (2005) 1.

[68] M. Guidal, M.V. Polayakov, A.V. Radyushkin, and M. Vanderhaeghen, Phys. Rev. D72, (2005) 054013. 